TRANSACTIONS OF THE

AMERICAN MATHEMATICAL SOCIETY

Volume 354, Number 8 , Pages 3181-3205

S 0002-9947(02)02997-5

Article electronically published on April 1, 2002

\title{
COMPACTNESS OF THE SOLUTION OPERATOR FOR A LINEAR EVOLUTION EQUATION WITH DISTRIBUTED MEASURES
}

\author{
IOAN I. VRABIE
}

\begin{abstract}
The main goal of the present paper is to define the solution operator $(\xi, g) \mapsto u$ associated to the evolution equation $d u=(A u) d t+d g$, $u(0)=\xi$, where $A$ generates a $C_{0}$-semigroup in a Banach space $X, \xi \in X$, $g \in B V([a, b] ; X)$, and to study its main properties, such as regularity, compactness, and continuity. Some necessary and/or sufficient conditions for the compactness of the solution operator extending some earlier results due to the author and to BARAS, HASSAN, Veron, as well as some applications to the existence of certain generalized solutions to a semilinear equation involving distributed, or even spatial, measures, are also included. Two concrete examples of elliptic and parabolic partial differential equations subjected to impulsive dynamic conditions on the boundary illustrate the effectiveness of the abstract results.
\end{abstract}

\section{INTRODUCTION}

Let $X$ be a Banach space, $A: D(A) \subseteq X \rightarrow X$ the infinitesimal generator of a $C_{0}$-semigroup of contractions, $\xi \in D(A)$, and let us consider the nonhomogeneous Cauchy problem

$$
\left\{\begin{array}{l}
u^{\prime}=A u+f \\
u(a)=\xi
\end{array}\right.
$$

This problem has a unique: (i) classical, or $C^{1}$ solution, if $f \in C^{1}[a, b]$; X) (see Corollary 2.5, p. 107 in PAZY [20]); (ii) strong solution, if $f$ is a.e. differentiable on $[a, b]$ and $f^{\prime} \in L^{1}(a, b ; X)$ (see Corollary 2.6, p. 108 in PAZY [20]); (iii) mild, or $C^{0}$ solution, if $f \in L^{1}(a, b ; X)$ ] There are however situations in which the "function" $f$ in the right-hand side does not satisfy even the minimal assumption to be in

Received by the editors March 19, 2001 and, in revised form, September 21, 2001.

2000 Mathematics Subject Classification. Primary 47D06, 46G10, 47B07; Secondary 35A05, 35J99, 35K99.

Key words and phrases. Linear evolution equation, $C_{0}$-semigroup, vector-valued function of bounded variation, compactness of the solution operator.

This research was supported in part by the CNCSU/CNFIS Grant C120(1998) of the World Bank and the Romanian Government.

${ }^{1}$ We recall that a mild, or $C^{0}$-solution of 1.1 is a continuous function $u:[a, b] \rightarrow X$ satisfying

$$
u(t)=S(t-a) \xi+\int_{a}^{t} S(t-s) f(s) d s
$$

for each $t \in[a, b]$, where $\{S(t) ; t \geq 0\}$ is the $C_{0}$-semigroup generated by $A$. So, in this case, both the existence and uniqueness are implicitly ensured by definition.

(C)2002 American Mathematical Society 
$L^{1}(a, b ; X)$. These situations arise, for instance, in optimal control problems with state constraints, when the so-called dual arc satisfies an equation of the type

$$
\left\{\begin{array}{l}
d p^{*}=\left(-A^{*} p^{*}+q\right) d t+d w \\
p^{*}(T)=p_{T}^{*}
\end{array}\right.
$$

where $A^{*}$ is the adjoint of $A, q \in L^{1}\left(0, T ; X^{*}\right)$, and $w:[0, T] \rightarrow X^{*}$ is a function of bounded variation. See Barbu, Precupanu [4, Definition 1.1, p. 258. From this reason at least, it is of interest to study the more general case (than (1.1)) of Cauchy problems of the type

$$
\left\{\begin{array}{l}
d u=(A u) d t+d g \\
u(a)=\xi
\end{array}\right.
$$

where $\xi \in X$, and $g:[a, b] \rightarrow X$ is a function of bounded variation. Roughly speaking, a solution of (1.2) is a piecewise continuous function $u$ defined by a Duhamel-like formula involving a Riemann-Stieltjes integral, i.e.,

$$
u(t)=S(t-a) \xi+\int_{a}^{t} S(t-s) d g(s) .
$$

See Definition 2.1 and Theorem 3.1 below. For earlier results concerning evolution equations whose solutions are defined via a quite similar variation of constants-like formula, for the specific case $d g(s)=f(s) d \nu(s)$, where $f \in L^{1}(a, b ; X)$ and $\nu$ is a bounded signed measure, see AHmed [1].

The main goal of the present paper is to study the fundamental properties of the solution operator $(\xi, g) \mapsto u$ associated to the Cauchy problem (1.2), such as regularity (Theorem 3.1), compactness (Theorem [5.2), and continuity (Theorem 7.1), and to extend to this general (but only linear) frame an earlier result due to the author referring to the mild solution operator associated to the classical Cauchy problem (1.1) with $A$ possibly nonlinear. See Theorem 6.1 and VRabie 28]. These kind of compactness properties are useful in establishing existence results for both Cauchy and periodic problems, as well as for optimal control problems, by means of topological arguments. See for instance Gutman [13], Hirano [15], 16], Hirano, Misoguchi [17, Pazy [19, Shioji 22], 23], Vrabie [24], 25], 26], 27], 28], and the references therein.

Our main result, Theorem 5.2 and its consequences, Theorem 6.2, which in its turn extends a known compactness result due to BARAs, Hassan, Veron [2], and Theorem 7.1, are the starting point in order to get information on the existence for certain abstract semilinear evolution equations involving highly irregular data, information which cannot be obtained via the classical compactness results as those used in the papers cited above. See Theorem 8.1, which may be considered as an extension of Pazy's main local existence result in 19 to the case of semilinear evolution equations governed by nonlinear distributed measure-valued perturbations of infinitesimal generators of $C_{0}$-semigroups, and its remarkable consequence, Theorem 8.2. In order to illustrate the power of the former theorem, we analyze two illuminating examples: one concerning a semilinear diffusion equation with impulses, and another concerning a semilinear elliptic problem, both subjected to impulsive dynamic conditions on the boundary.

The interesting feature of our main result consists in showing that a quite natural compactness condition on the images suffices to ensure the $L^{p}$-equi-integrability of a family of solutions for (1.2) corresponding to bounded data, and therefore its 
relative compactness in $L^{p}(a, b ; X)$. Surprisingly, the $L^{p}$-equi-integrability condition is not automatically satisfied by any family of solutions, also corresponding to bounded data, as suggested by finite-dimensional examples, but only by those just mentioned, i.e., enjoying some additional compactness conditions. See Example 5.1

The paper is divided into nine sections, the first four being concerned with such basic properties of the solutions as continuity outside of the support of the singular part of $\operatorname{Var}(g,[a, \cdot])$, right (left) continuity at those points at which $g$ is continuous from the right (left), relationships with other types of solutions, etc. Section 5 contains the statement and proof of our main result, while section 6 is concerned with some of its consequences. Section 7 includes some facts referring to abstract evolution equations with "spatial" distributed measures as data, answering a question rised by BARBU [3, and is somehow related to some specific existence results in BrÉzis, Friedman [8. Section 8 presents an abstract existence theorem referring to a class of abstract semilinear evolution equations involving distributed measures, while the last Section 9 contains two significant examples of partial differential equations showing how the abstract theory applies.

Acknowledgements. The author takes this opportunity to express his warmest thanks to Professor Ştefan Frunză for fruitful discussions and criticism, as well as to Dr. Corneliu Ursescu, Senior Researcher at the Mathematics Institute of the Romanian Academy, for a very careful reading of the manuscript.

\section{Preliminaries}

We begin by giving a precise sense in which we have to understand the relations (1.2). Let $\mathcal{D}[a, b]$ be the set of all partitions of the interval $[a, b]$. We recall that, if $g:[a, b] \rightarrow X$, then, for each $\Delta \in \mathcal{D}[a, b], \Delta: a=t_{0}<t_{1}<\cdots<t_{k}=b$, the number

$$
\operatorname{Var}_{\Delta}(g,[a, b])=\sum_{i=0}^{k-1}\left\|g\left(t_{i+1}\right)-g\left(t_{i}\right)\right\|
$$

is called the variation of the function $g$ relative to the partition $\Delta$. If

$$
\sup _{\Delta \in \mathcal{D}[a, b]} \operatorname{Var}_{\Delta}(g,[a, b])<\infty,
$$

then $g$ is said to be of bounded variation, and the number

$$
\operatorname{Var}(g,[a, b])=\sup _{\Delta \in \mathcal{D}[a, b]} \operatorname{Var}_{\Delta}(g,[a, b])
$$

is called the variation of the function $g$ on the interval $[a, b]$. Similarly, we define $\operatorname{Var}(g,(a, b]), \operatorname{Var}(g,(a, b))$, and $\operatorname{Var}(g,[a, b))$. We notice that, in each of the above three cases, we have to consider only those partitions $\Delta$ satisfying, either $a<t_{0}$, or $t_{k}<b$, or both, as required by the type of the interval considered. As usual, we denote by $B V([a, b] ; X)$ the vector space of all functions of bounded variation from $[a, b]$ to $X$. The mapping $g \mapsto \operatorname{Var}(g,[a, b])$ is a seminorm on $B V([a, b] ; X)$, while $g \mapsto\|g(a)\|+\operatorname{Var}(g,[a, b])$ is a norm, denoted by $\|\cdot\|_{B V([a, b] ; X)}$, and in respect to which $B V([a, b] ; X)$ is a Banach space.

\footnotetext{
${ }^{2}$ Some authors, as for instance Hille, Phillips [14], p. 59, call such functions functions of strong bounded variation.
} 
For details on infinite-dimensional vector-valued functions of bounded variation, whose systematic study was initiated by Bochner, TAYLor [6], see BARBU, PreCUPANU [4], BRÉzIS [7, and HÖNIG [18. We assume familiarity with the basic concepts and results concerning $C_{0}$-semigroups and linear evolution equations in general Banach spaces. For details, we refer the reader to Engel, NAgel [11, and PAZY [20].

Let $\{S(t) ; t \geq 0\}$ be a $C_{0}$-semigroup of contractions on $X$, let $\left\{S(t)^{*} ; t \geq 0\right\}$ be the dual semigroup defined on $X^{*}$, and $\left\{S(t)^{\odot} ; t \geq 0\right\}$ the sun dual semigroup. We recall that $S(t)^{\odot}: X^{\odot} \rightarrow X^{\odot}$ is defined by

$$
X^{\odot}=\left\{x^{*} \in X^{*} ; \lim _{h \downarrow 0}\left\|S(h)^{*} x^{*}-x^{*}\right\|=0\right\},
$$

and

$$
S(t)^{\odot} x^{\odot}=S(t)^{*} x^{\odot}
$$

for each $x^{\odot} \in X^{\odot}$. It is know that $X^{\odot}$ is a closed subspace of $X^{*}$. See Hille, Phillips [14, Theorem 14.2.1, p. 422. Also from Theorem 14.2.1 (loc.cit.) it follows that $X^{\odot}$ is weakly star dense in $X^{*}$, or equivalently, for each $x \in X$, we have

$$
\|x\|=\sup \left\{\left|\left(x, x^{\odot}\right)\right| ; x^{\odot} \in X^{\odot},\left\|x^{\odot}\right\| \leq 1\right\} .
$$

Let $t \in(a, b], \Delta \in \mathcal{D}[a, t], \Delta: a=t_{0}<t_{1}<\cdots<t_{k}=t$, let $\tau_{i} \in\left[t_{i}, t_{i+1}\right]$, $i=0,1, \ldots, k-1$, and consider the Riemann-Stieltjes sum of $\tau \mapsto S(t-\tau)$ over $[a, t]$ with respect to $g$, i.e.

$$
\sigma_{[a, t]}\left(\Delta, S, g, \tau_{i}\right)=\sum_{i=0}^{k-1} S\left(t-\tau_{i}\right)\left(g\left(t_{i+1}\right)-g\left(t_{i}\right)\right) .
$$

If $\Delta$ is a partition of $[a, t]$, we denote $\lambda(\Delta)=\max \left\{t_{i+1}-t_{i} ; i=0,1, \ldots, k-1\right\}$. Using the very same arguments as in the scalar case, we easily deduce that, for each sequence $\left(\Delta_{n}\right)_{n}$ with $\lim _{n \rightarrow \infty} \lambda\left(\Delta_{n}\right)=0$, each intermediate points $\tau_{i}^{(n)}$ in $\left[t_{i}^{(n)}, t_{i+1}^{(n)}\right], i=0,1, \ldots, k_{n}-1$, and each $x^{\odot} \in X^{\odot},\left(\left(\sigma_{[a, t]}\left(\Delta_{n}, S, g, \tau_{i}^{(n)}\right), x^{\odot}\right)\right)_{n}$ is a Cauchy sequence. Let $X^{c}$ be the sequential completion of $X$ in the $\sigma\left(X, X^{\odot}\right)$ topology ${ }^{3}$, i.e. the space of all elements $x^{c} \in X^{* *}$ for which there exists a weakly- $\odot$ Cauchy sequence $\left(x_{k}\right)_{k}$ in $X$ such that, for each $x^{\odot} \in X^{\odot}$, we have $\left(x^{\odot}, x^{c}\right)=$ $\lim _{k}\left(x_{k}, x^{\odot}\right)$. Endowed with the usual sup-norm, i.e.,

$$
\left\|x^{c}\right\|=\sup \left\{\left|\left(x^{\odot}, x^{c}\right)\right| ; x^{\odot} \in X^{\odot},\left\|x^{\odot}\right\| \leq 1\right\},
$$

$X^{c}$ is a norm closed subspace of $X^{* *}$. The arguments above show that there exists a unique element $\int_{a}^{t} S(t-s) d g(s) \in X^{c}$ such that

$$
\int_{a}^{t} S(t-s) d g(s)=\lim _{\lambda(\Delta) \downarrow 0} \sum_{i=0}^{k-1} S\left(t-\tau_{i}\right)\left(g\left(t_{i+1}\right)-g\left(t_{i}\right)\right)
$$

weakly- $\odot$. This is called the Riemann-Stieltjes integral on $[a, t]$ of the operatorvalued function $\tau \mapsto S(t-\tau)$ with respect to the vector-valued function $g$. If $\alpha:[a, b] \rightarrow \mathbb{R}$ is a given function, by a similar procedure, we can define

$$
\int_{a}^{t} \alpha(s) S(t-s) d g(s)=\lim _{\lambda(\Delta) \downarrow 0} \sum_{i=0}^{k-1} \alpha\left(\tau_{i}\right) S\left(t-\tau_{i}\right)\left(g\left(t_{i+1}\right)-g\left(t_{i}\right)\right),
$$

\footnotetext{
${ }^{3}$ From here on, we shall call this the weak- $\odot$ topology on $X$.
} 
of course, whenever the limit on the right-hand side exists in the weak- $\odot$ topology on $X$. It it easy to see that this happens, for instance, if $\alpha$ is the characteristic function of a proper subinterval of $[a, t]$.

Remark 2.1. A simple computational argument shows that, for each $[c, d] \subseteq[a, b]$ and each $t \in[d, b]$, we have

$$
\int_{c}^{d} S(t-s) d g(s)=\int_{c}^{d} \chi_{(c, d]}(s) S(t-s) d g(s)+S(t-c)(g(c+0)-g(c)),
$$

where $\chi_{(c, d]}$ denotes the characteristic function of the interval $(c, d]$. Similarly, for each $[c, d] \subseteq[a, b]$ and each $t \in[d, b]$, we have

$$
\int_{c}^{d} S(t-s) d g(s)=\int_{c}^{d} \chi_{[c, d)}(s) S(t-s) d g(s)+S(t-d)(g(d)-g(d-0)) .
$$

Remark 2.2. If $X$ is reflexive, the weak- $\odot$ topology on $X$ is nothing else than the weak topology on $X$, and therefore $X^{c}=X$. In general this is not the case, as the following simple example shows.

Example 2.1. Let $X=L^{1}(\mathbb{R})$, and let $\{S(t) ; t \geq 0\}$ be the $C_{0}$-group of translations, i.e.

$$
(S(t) f)(x)=f(x+t)
$$

for each $f \in X$, each $t \in \mathbb{R}$, and a.e. for $x \in \mathbb{R}$. It is well known that, in this case, $X^{\odot}=C_{u b}(\mathbb{R})$, i.e., the space of all bounded, uniformly continuous functions on $\mathbb{R}$, endowed with the usual sup-norm. See Engel, NAgel [11, Examples, (i), p. 63. At this point let us observe that the weak topology on $X$ does not coincide with the weak- $\odot$ topology. More than this, $X$ is not weakly- $\odot$ sequentially complete, and its sequential completion is a space of measures strictly larger that $X$. One may easily verify that all Dirac measures belong to $X^{c}$.

Remark 2.3. If $g$ is defined by a density, i.e. there exists $f \in L^{1}(a, b ; X)$ such that $d g(s)=f(s) d s$, one may prove that, for each $t \in[a, b]$, the limit in (2.1) exists in the norm topology of $X$, and

$$
\int_{a}^{t} S(t-s) d g(s)=\int_{a}^{t} S(t-s) f(s) d s \in X .
$$

This happens, for example, whenever $X$ has the Radon-Nicodým property (see Diestel, Uhl [9], Definition 3, p. 61), and $g$ is absolutely continuous on $[a, b]$, in which case $f=g^{\prime}$ a.e. on $[a, b]$. Some specific but important such instances are those in which $X$ is either reflexive, or a separable dual. See also Diestel, Uhu [9, Theorem 1, p. 79, and Corollary 4, p. 82.

Remarks 2.2 and 2.3 show that, in order that the Riemann-Stieltjes integral defined as above belong to $X$, we must impose some extra conditions on $X$, on $g$, or on $A$. Since in the applications we have in mind $X$ is a space enjoying quite bad geometric properties, as $L^{1}(\Omega)$ for instance, and thus nonreflexive, and $g$ is not a priori known because it comes by a passing to the limit process in a sequence of $L^{1}$-functions with respect to some weak topology which excludes the situation in Remark [2.3, in that follows, we will mainly focus our attention only on the properties of $A$ which may ensure the existence of a "good integral". The next theorem gives a useful sufficient condition in this respect. For the sake of completeness we first recall the following slight extension of Lemma 16 on p. 140 
in Dunford, Schwartz [10]. Since its proof follows exactly the same lines as the proof of the just mentioned Lemma 16, we do not enter into details.

Lemma 2.1. For each $g \in B V([a, b] ; X)$ and $t \in[a, b)$, respectively $s \in(a, b]$, we have

$$
\lim _{h \downarrow 0} \operatorname{Var}(g,(t, t+h])=0
$$

and respectively

$$
\lim _{h \downarrow 0} \operatorname{Var}(g,[s-h, s))=0 .
$$

The simple remark below will prove useful in what follows.

Remark 2.4. If $g \in B V([a, b] ; X)$, then $g([a, b])$ is strongly relatively compact in $X$. This is an easy consequence of the fact that $g$ is piecewise continuous on $[a, b]$, i.e. it has one-sided limits at each point in $[a, b]$.

Theorem 2.1. If the semigroup generated by $A$ is continuous from $(0,+\infty)$ to $\mathcal{L}(X)$ in the uniform operator topology, then, for each $g \in B V([a, b] ; X)$ and each $t \in(a, b)$, the limit in (2.1) exists in the norm topology of $X$, and consequently we have

$$
\int_{a}^{t} S(t-s) d g(s) \in X
$$

Proof. It suffices to show that, for some fixed $\ell>0$, for each $t \in(a, b]$, and each $\varepsilon>0$, there exists $\eta(\varepsilon)>0$ such that

$$
\left\|\sigma_{[a, t]}\left(\Delta, S, g, \tau_{i}\right)-\sigma_{[a, t]}\left(\Delta^{\prime}, S, g, \tau_{j}^{\prime}\right)\right\| \leq \ell \varepsilon
$$

provided $\lambda(\Delta) \leq \eta(\varepsilon)$ and $\Delta^{\prime}$ is finer than $\Delta$, i.e., contains all the points of $\Delta$. To fix the notation, let $\Delta \in \mathcal{D}[a, t], \Delta: a=t_{0}<t_{1}<\cdots<t_{k}=t$, be a partition of $[a, t]$, and let $\tau_{i}$ be arbitrary in $\left[t_{i}, t_{i+1}\right], i=0,1, \ldots, k-1$. If $\Delta^{\prime}$ is another partition of $[a, t]$ which is finer that $\Delta$, relabelling if necessary, we have $\Delta^{\prime}: t_{i}=t_{i, 0} \leq \tau_{i, 0} \leq t_{i, 1} \leq \tau_{i, 1} \leq \cdots \leq \tau_{i, m_{i}-1} \leq t_{i, m_{i}}=t_{i+1}, i=0,1, \ldots, k-1$. Let $\varepsilon>0$, and fix $\delta>0$ with $t-2 \delta \in[a, b]$ and such that

$$
\left\|(S(s)-I)\left(g(\tau)-g\left(\tau^{\prime}\right)\right)\right\| \leq \varepsilon
$$

for each $s \in[0, \delta]$ and each $\tau, \tau^{\prime} \in[a, t)$, and

$$
\operatorname{Var}(g,[t-2 \delta, t)) \leq \varepsilon .
$$

This is always possible, because the semigroup is strongly continuous, $g([a, t))$ is strongly relatively compact in $X$ (see Remark 2.4), while, by virtue of Lemma 2.1 $\lim _{\delta \downarrow 0} \operatorname{Var}(g,[t-\delta, t))=0$. Since $\tau \mapsto S(\tau)$ is continuous at $\tau=\delta>0$ in the uniform operator topology, there exists $\eta \in(0, \delta]$ such that

$$
\|S(s) S(\delta)-S(\delta)\| \leq \varepsilon
$$

for each $s \in[0, \eta]$.

Let us assume now that $\lambda(\Delta) \leq \eta$, and let us fix $p=p(\delta) \in\{1,2, \ldots, k-1\}$ such that $t_{p}<t-\delta$ and $t_{p+1} \geq t-\delta$. Let us denote

$$
S_{n}^{h}=\sum_{i=n}^{h-1} \sum_{j=0}^{m_{i}-1}\left(S\left(t-\tau_{i}\right)-S\left(t-\tau_{i, j}\right)\right)\left(g\left(t_{i, j+1}\right)-g\left(t_{i, j}\right)\right),
$$


and let us observe that

$$
\sigma_{[a, t]}\left(\Delta, S, g, \tau_{i}\right)-\sigma_{[a, t]}\left(\Delta^{\prime}, S, g, \tau_{i, j}\right)=S_{0}^{k} .
$$

Set $S_{0}^{k}=T_{1}+T_{2}+T_{3}$, where $T_{1}=S_{0}^{p}, T_{2}=S_{p}^{k}-T_{3}$, and $T_{3}$ is the last term in $S_{0}^{k}$. By (2.7) and the fact that $\delta<t-t_{p}$, we deduce that

$$
\begin{aligned}
& \left\|T_{1}\right\| \leq \sum_{i=0}^{p-1} \sum_{j=0}^{m_{i}-1}\left\|\left[S\left(t_{p}-\tau_{i}\right)-S\left(t_{p}-\tau_{i, j}\right)\right] S\left(t-t_{p}\right)\left(g\left(t_{i, j+1}\right)-g\left(t_{i, j}\right)\right)\right\| \\
& \leq \sum_{i=0}^{p-1} \sum_{j=0}^{m_{i}-1}\left\|S\left(\left|\tau_{i}-\tau_{i, j}\right|\right) S(\delta)-S(\delta)\right\|\left\|g\left(t_{i, j+1}\right)-g\left(t_{i, j}\right)\right\| \leq \operatorname{Var}(g,[a, b]) \varepsilon .
\end{aligned}
$$

Since $t_{p+1}-t_{p} \leq \eta, t_{k-1, m_{k-1}-1}-t_{p+1} \leq t-t_{p+1} \leq \delta$, and $\eta \in(0, \delta]$, in view of (2.6), we get

$$
\left\|T_{2}\right\| \leq 2 \operatorname{Var}(g,[t-\eta-\delta, t)) \leq 2 \operatorname{Var}(g,[t-2 \delta, t)) \leq 2 \varepsilon .
$$

Finally, by virtue of (2.5), we have

$$
\left\|T_{3}\right\| \leq\left\|\left(S\left(\left|\tau_{k}-\tau_{k-1, m_{k-1}-1}\right|\right)-I\right)\left(g(t)-g\left(t_{k-1, m_{k-1}-1}\right)\right)\right\| \leq \varepsilon .
$$

Consequently

$$
\left\|\sigma_{[a, t]}\left(\Delta, S, g, \tau_{i}\right)-\sigma_{[a, t]}\left(\Delta^{\prime}, S, g, \tau_{i, j}\right)\right\| \leq(\operatorname{Var}(g,[a, b])+3) \varepsilon,
$$

and this completes the proof.

Definition 2.1. Let $\xi \in X$ and $g \in B V([a, b] ; X)$. A function $u:[a, b] \rightarrow X^{c}$, defined by

$$
u(t)=S(t-a) \xi+\int_{a}^{t} S(t-s) d g(s)
$$

for each $t \in[a, b]$, and satisfying (2.4), is called the $\mathcal{L}^{\infty}$-solution of the problem (1.2) on the interval $[a, b]$ with the initial condition $u(a)=\xi$.

Remark 2.5. If $x \in X$ and $\lim _{n} x_{n}=x$ weakly- $\odot$ in $X$, then

$$
\|x\| \leq \liminf _{n}\left\|x_{n}\right\|
$$

Consequently, whenever $\int_{a}^{t} S(t-s) d g(s) \in X$, we have

$$
\left\|\int_{a}^{t} S(t-s) d g(s)\right\| \leq \int_{a}^{t} d \operatorname{Var}(g,[a, s)]=\operatorname{Var}(g,[a, t]) .
$$

Remark 2.6. Clearly, whenever $d g$ is defined by means of a density $f$, i.e. $d g=f d t$ with $f \in L^{1}(a, b ; X)$, the $\mathcal{L}^{\infty}$-solution of (1.2) corresponding to $(\xi, g)$ is nothing else than the mild, or $C^{0}$-solution of (1.1) corresponding to $(\xi, f)$. See Remark 2.3

The proofs of the next two propositions follow from an elementary computational argument, and therefore we do not give details.

Proposition 2.1 (Concatenation Principle). Let $v:[a, c] \rightarrow X$ be the $\mathcal{L}^{\infty}$-solution of the problem (1.2) on the interval $[a, c]$ with the initial condition $v(a)=\xi$, and $w:[c, b] \rightarrow X$ the $\mathcal{L}^{\infty}$-solution of the very same problem on $[c, b]$ with the initial condition $w(c)=v(c)$. Then, the function $u:[a, b] \rightarrow X$, defined by

$$
u(t)= \begin{cases}v(t) & \text { if } t \in[a, c], \\ w(t) & \text { if } t \in(c, b]\end{cases}
$$


is the $\mathcal{L}^{\infty}$-solution of the problem (1.2) on the interval [a,b], with the initial datum $u(a)=\xi$.

The concatenation principle is equivalent to the fact that the family of mappings $\{U(t, s) ; a \leq s \leq b\} \in \mathcal{L}(X)$, defined by $U(t, s) \xi=S(t-s) \xi+\int_{s}^{t} S(t-\tau) d g(\tau)$ for each $\xi \in X$, satisfies the following properties of an evolution system: $U(s, s)=I$ and $U(t, s)=U(t, \tau) U(\tau, s)$ for each $a \leq s \leq \tau \leq t \leq b$, and this follows from the simple proposition below.

Proposition 2.2 (Evolution Property). If $u:[a, b] \rightarrow X$ is the $\mathcal{L}^{\infty}$-solution of the problem (1.2), then, for each $c \in(a, b)$, we have

$$
u(t)=S(t-c) u(c)+\int_{c}^{t} S(t-s) d g(s) .
$$

\section{Regularity of $\mathcal{L}^{\infty}$-SOlutions}

We begin with the following fundamental regularity result.

Theorem 3.1. Let $\xi \in X, g \in B V([a, b] ; X)$, and let $u$ be the $\mathcal{L}^{\infty}$-solution of (1.2) corresponding to $\xi$ and $g$. Then, for each $t \in[a, b)$ and each $s \in(a, b]$, we have

$$
\begin{gathered}
u(t+0)-u(t)=g(t+0)-g(t), \\
u(s)-u^{*}(s-0)=g(s)-g(s-0),
\end{gathered}
$$

where

$$
u^{*}(s-0)=\lim _{h \downarrow 0} S(h) u(s-h) .
$$

If, in addition, either the semigroup generated by $A$ is continuous from $(0,+\infty)$ to $\mathcal{L}(X)$ in the uniform operator topology, or it can be embedded into a group, then $u^{*}(s-0)=u(s-0)$, and accordingly

$$
u(s)-u(s-0)=g(s)-g(s-0) .
$$

So, in this case, $u$ is continuous from the right (left) at $t \in[a, b]$ if and only if $g$ is continuous from the right (left) at $t$. In particular, $u$ is continuous at any point at which $g$ is continuous, and thus $u$ is piecewise continuous on $[a, b]$.

Proof. Since $u$ satisfies the evolution property (see Proposition 2.2) and, for each $t \in[a, b]$ and $h>0$ with $t+h \leq b$, we have

$$
\begin{gathered}
u(t+h)-u(t)=S(h) u(t)+\int_{t}^{t+h} S(t+h-s) d g(s)-u(t) \\
=S(h) u(t)-u(t)+\int_{t}^{t+h} \chi_{(t, t+h]}(s) S(t+h-s) d g(s)+S(h)(g(t+0)-g(t)),
\end{gathered}
$$

by virtue of Remark 2.5, we get

$$
\begin{aligned}
\| u(t+h) & -u(t)-g(t+0)+g(t) \| \\
\leq & \|S(h)(u(t)+g(t+0)-g(t))-u(t)-g(t+0)+g(t)\| \\
& +\operatorname{Var}(g,(t, t+h]) .
\end{aligned}
$$


From the strong continuity of the semigroup and Lemma 2.1, we deduce (3.1). To check (3.2), let $s \in(a, b]$, and $h>0$ with $s-h \geq a$, and let us observe that, by using similar arguments, we obtain

$$
\begin{gathered}
u(s)=S(h) u(s-h)+\int_{s-h}^{s} S(s-\tau) d g(\tau) \\
=S(h) u(s-h)+\int_{s-h}^{s} \chi_{[s-h, s)}(\tau) S(s-\tau) d g(\tau)+g(s)-g(s-0),
\end{gathered}
$$

and therefore

$$
\|u(s)-S(h) u(s-h)-g(s)+g(s-0)\| \leq \operatorname{Var}(g,[s-h, s)) .
$$

An appeal to Lemma 2.1 completes the proof of (3.2). Let us assume next that the semigroup $\{S(t) ; t \geq 0\}$ is continuous in the uniform operator topology from $(0,+\infty)$ to $\mathcal{L}(X)$. Let $s \in(a, b], \delta>0$ with $s-\delta \in[a, b], h \in(0, \delta]$, and denote $\psi(s, h, \tau)=S(s-\tau)-S(s-h-\tau)$. We have

$$
\|u(s-h)-S(h) u(s-h)\| \leq\|S(h) \xi-\xi\|+\left\|\int_{a}^{s-h} \psi(s, h, \tau) d g(\tau)\right\| .
$$

Since the semigroup is strongly continuous at 0 , to complete the proof, it suffices to show that the second term on the right-hand side tends to 0 when $h$ tends to 0 . Let $\varepsilon>0$. By virtue of Lemma [5.1, there exists $\delta>0$ such that

$$
\operatorname{Var}(g,[s-\delta, s)) \leq \varepsilon
$$

So, in view of (2.3) and (2.9), for each $h \in(0, \delta)$, we get

$$
\begin{gathered}
\left\|\int_{a}^{s-h} \psi(s, h, \tau) d g(\tau)\right\| \leq\left\|\int_{a}^{s-\delta} \psi(s, h, \tau) d g(\tau)\right\|+\left\|\int_{s-\delta}^{s-h} \psi(s, h, \tau) d g(\tau)\right\| \\
\leq\|S(\delta)-S(\delta-h)\| \operatorname{Var}(g,[a, b])+2 \operatorname{Var}(g,[s-\delta, s-h)) \\
+\|(S(h)-I)(g(s-h)-g(s-h-0))\| \leq\|S(\delta)-S(\delta-h)\| \operatorname{Var}(g,[a, b]) \\
+2 \operatorname{Var}(g,[s-\delta, s))+\|(S(h)-I)(g(s-h)-g(s-h-0))\| .
\end{gathered}
$$

Since the semigroup is continuous in the uniform operator topology from $(0,+\infty)$ to $\mathcal{L}(X)$ and, by Remark $2.4 g([a, b])$ is relatively compact in $X$, for the very same $\varepsilon>0$, there exists $\eta \in(0, \delta)$ such that we have both

$$
\sup _{\theta \in(0, \eta)}\|S(\delta)-S(\delta-\theta)\| \leq \varepsilon
$$

and

$$
\|(S(h)-I)(g(s-h)-g(s-h-0))\| \leq \varepsilon
$$

for each $h \in(0, \eta)$. Summing up, we get

$$
\left\|\int_{a}^{s-h} \psi(s, h, \tau) d g(\tau)\right\| \leq(\operatorname{Var}(g,[a, b])+2) \varepsilon,
$$

which shows that $\lim _{h \downarrow 0}\|u(s-h)-S(h) u(s-h)\|=0$. Recalling that, as we already have shown, there exists $\lim _{h \downarrow 0} S(h) u(s-h)=u^{*}(s-0)$, we conclude that $u(s-0)$ exists and $u(s-0)=u^{*}(s-0)$. This proves (3.3) in the case in which the 
semigroup is continuous in the uniform operator topology on $(0,+\infty)$. Finally, if $\{S(t) ; t \geq 0\}$ can be embedded into a group, for each $t \geq 0, S(t)$ is invertible, and so

$$
u(t-h)=S(h)^{-1} S(h) u(t-h) .
$$

Thus, by virtue of (3.2) and the strong continuity of both $S(h)$ and $S(h)^{-1}$, we get (3.3). The proof is complete.

Remark 3.1. It is a simple exercise to show that, even for general $C_{0}$-semigroups, the $\operatorname{limit}_{h \downarrow 0} u(s-h)$ exists in the weak- $\odot$ topology on $X$ and equals $u^{*}(s-0)$. So, we have

$$
u(s)-u(s-0)=g(s)-g(s-0)
$$

for each $s \in(a, b]$, where $u(s-0)$ is considered in the weak- $\odot$ topology on $X$.

Corollary 3.1. Let $g \in B V([a, b] ; X)$. If $g$ is right continuous on $[a, b]$, then, for each $\xi \in X$, the $\mathcal{L}^{\infty}$-solution of (1.2) corresponding to $\xi$ and $g$ is right continuous on $[a, b]$. If either the semigroup generated by $A$ is continuous from $(0,+\infty)$ to $\mathcal{L}(X)$ in the uniform operator topology, or it can be embedded into a group and $g \in B V([a, b] ; X)$ is continuous, then, for each $\xi \in X$, the $\mathcal{L}^{\infty}$-solution of (1.2) corresponding to $\xi$ and $g$ is continuous on $[a, b]$.

Remark 3.2. A quite natural question we may raise is whether or not any $\mathcal{L}^{\infty}$ solution is of bounded variation. The answer to this question is in the negative, as we can see from the next simple example. Let $X$ be a reflexive Banach space, and let us assume that the semigroup generated by $A$ is not differentiable, i.e. there exists $x \in X$ such that $S(t) x \notin D(A)$ for each $t \geq 0$. 4 Now, let $g:[0,2] \rightarrow X$ be defined by

$$
g(t)= \begin{cases}0 & \text { for } t \in[0,1) \\ x & \text { for } t \in[1,2]\end{cases}
$$

Clearly $g$ is of bounded variation on $[1,2]$. Moreover one may easily see that the unique $\mathcal{L}^{\infty}$-solution $u$ of the problem (1.2) corresponding to $\xi=0$ and to $g$ is defined by

$$
u(t)=\left\{\begin{array}{cc}
0 & \text { for } t \in[0,1), \\
S(t-1) x & \text { for } t \in[1,2]
\end{array}\right.
$$

At this point let us recall that, whenever $X$ is reflexive and $u:[a, b] \rightarrow X$ is of bounded variation, then $u$ is a.e. differentiable on $[a, b]$. See Bochner, TAYLOR [6], Theorem 5.2. From this remark it is clear that $u$ cannot be of bounded variation on $[0,2]$, since it is nowhere differentiable on $[1,2]$. We conclude this remark by mentioning that it should be of great interest to know under what circumstances on $X, \xi, A$, and $g$ the corresponding $\mathcal{L}^{\infty}$-solution of (1.2) is of bounded variation. One may prove that this is the case whenever $A \in \mathcal{L}(X)$, i.e. when the generated semigroup is uniformly continuous, but we don't know any other relevant situations.

\footnotetext{
${ }^{4}$ A typical instance of this sort is $X=L^{2}\left(\mathbb{R}_{+} ; \mathbb{R}\right)$ and $A: D(A) \subseteq X \rightarrow \mathrm{X}$ defined by $A f=$ $f^{\prime}$, for each $f \in D(A)=\left\{f \in L^{2}\left(\mathbb{R}_{+} ; \mathbb{R}\right) ; f^{\prime} \in L^{2}\left(\mathbb{R}_{+} ; \mathbb{R}\right)\right\}$, which generates the well-known translation semigroup.
} 


\section{A Characterization of $\mathcal{L}^{\infty}$-SOlutions}

In this section we prove a characterization of $\mathcal{L}^{\infty}$-solutions in terms of the duality between $X$ and $X^{c}$. More precisely, let $A^{\odot}: D\left(A^{\odot}\right) \subseteq X^{\odot} \rightarrow X^{\odot}$ be the infinitesimal generator of the sun dual semigroup $\left\{S^{\odot}(t) ; t \geq 0\right\}$. We introduce:

Definition 4.1. A function $u:[a, b] \rightarrow X$ is called a variational, or $\mathcal{V}$-solution of the problem (1.2) if $u$ satisfies (3.1) and (3.4) and, for each $f^{\odot} \in C\left([a, b] ; D\left(A^{\odot}\right)\right)$, we have

$$
\int_{a}^{b}\left(u(t), f^{\odot}(t)\right) d t+\int_{a}^{b}\left(d g(t), \varphi^{\odot}(t)\right)+\left(\xi, \varphi^{\odot}(a)\right)=0,
$$

where $\varphi^{\odot} \in C\left([a, b] ; D\left(A^{\odot}\right)\right) \cap C^{1}\left([a, b] ; X^{\odot}\right)$ is the unique strong solution of the $\odot$-adjoint problem

$$
\left\{\begin{array}{l}
\varphi^{\odot}=-A^{\odot} \varphi^{\odot}+f \odot \\
\varphi^{\odot}(b)=0
\end{array}\right.
$$

and the middle term on the left-hand side of (4.1) is the Riemann-Stieltjes integral of the function $\varphi^{\odot}$ with respect to $g$, i.e.

$$
\int_{a}^{b}\left(d g(t), \varphi^{\odot}(t)\right)=\lim _{\lambda(\Delta) \downarrow 0} \sum_{i=0}^{k-1}\left(g\left(t_{i+1}\right)-g\left(t_{i}\right), \varphi^{\odot}\left(\tau_{i}\right)\right) .
$$

Clearly each $\mathcal{V}$-solution of (1.2) is weakly- $\odot$ piecewise continuous on $[a, b]$. Moreover, since $D\left(A^{\odot}\right)$ is dense in $X^{\odot}$, it follows that $C\left([a, b] ; D\left(A^{\odot}\right)\right)$ is dense in $C\left([a, b] ; X^{\odot}\right)$ too, and accordingly we have

Theorem 4.1. For each $\xi \in X$ and each $g \in B V([a, b] ; X)$, the problem (1.2) has at most one $\mathcal{V}$-solution defined on $[a, b]$.

Proof. The conclusion follows from the simple remark that, whenever $u$ and $v$ are two $\mathcal{V}$-solutions of (1.2), $u-v$ is weakly- $\odot$ continuous on $[a, b]$ (see (3.1) and (3.4)), and

$$
\int_{a}^{b}\left(u(t)-v(t), f^{\odot}(t)\right) d t=0
$$

for each $f \in C\left([a, b] ; X^{\odot}\right)$.

As for the $\mathcal{V}$-solution, we have the following characterization theorem.

Theorem 4.2. Let $\xi \in X$ and $g \in B V([a, b] ; X)$. Then $u \in \mathcal{L}^{\infty}(a, b ; X)$ is the $\mathcal{V}$-solution of the problem (1.2) on $[a, b]$ if and only if $u$ is the $\mathcal{L}^{\infty}$-solution of the same problem on the same interval.

Proof. Let $u$ be an $\mathcal{L}^{\infty}$-solution of (1.2). Clearly $u$ satisfies (3.1) and (3.4). So, by virtue of the Uniqueness Theorem 4.1, it suffices to verify (4.1). We have

$$
\begin{gathered}
\int_{a}^{b}\left(u(t), f^{\odot}(t)\right) d t+\int_{a}^{b}\left(d g(t), \varphi^{\odot}(t)\right)+\left(\xi, \varphi^{\odot}(a)\right) \\
=\int_{a}^{b}\left(S(t-a) \xi, f^{\odot}(t)\right) d t+\int_{a}^{b}\left(\int_{a}^{t} S(t-s) d g(s), f^{\odot}(t)\right) d t \\
+\int_{a}^{b}\left(d g(t), \varphi^{\odot}(t)\right)+\left(\xi, \varphi^{\odot}(a)\right) .
\end{gathered}
$$


Let us observe that, for each $t, s \in[a, b]$, we have $\chi_{[a, t]}(s)=\chi_{[s, b]}(t)$, where $\chi_{E}$ is the characteristic function of the subset $E \subseteq[a, b]$, and therefore

$$
\begin{gathered}
\int_{a}^{b}\left(\int_{a}^{t} S(t-s) d g(s), f^{\odot}(t)\right) d t=\int_{a}^{b}\left(\int_{a}^{b} \chi_{[a, t]}(s) S(t-s) d g(s), f^{\odot}(t)\right) d t \\
=\int_{a}^{b}\left(\int_{a}^{b} \chi_{[s, b]}(t) S(t-s) d g(s), f^{\odot}(t)\right) d t \\
=\int_{a}^{b}\left(d g(s), \int_{a}^{b} \chi_{[s, b]}(t) S^{\odot}(t-s) f^{\odot}(t) d t\right) \\
=\int_{a}^{b}\left(d g(s), \int_{s}^{b} S^{\odot}(t-s) f^{\odot}(t) d t\right)
\end{gathered}
$$

We also have

$$
\begin{aligned}
& \qquad \int_{a}^{b}\left(S(t-a) \xi, f^{\odot}(t)\right) d t=\int_{a}^{b}\left(\xi, S^{\odot}(t-a) f^{\odot}(t)\right) d t \\
& \text { (4.4) } \varphi^{\odot}(s)=-\int_{s}^{b} S^{\odot}(t-s) f^{\odot}(t) d t \text { and } \varphi^{\odot}(a)=-\int_{a}^{b} S^{\odot}(t-a) f^{\odot}(t) d t . \\
& \text { From these relations and (4.3), we deduce (4.1). }
\end{aligned}
$$

Conversely, if $u$ is a $\mathcal{V}$-solution of (1.2) on $[a, b]$ and $f^{\odot} \in C\left([a, b] ; X^{\odot}\right)$, then $\varphi^{\odot}$ satisfies (4.4), and a simple backward calculation shows that (4.1) implies

$$
\int_{a}^{b}\left(u(t)-S(t-a) \xi-\int_{a}^{t} S(t-s) d g(s), f^{\odot}(t)\right) d t=0 .
$$

The conclusion follows from the arbitrariness of $f^{\odot} \in C\left([a, b] ; X^{\odot}\right)$ combined with the fact that, by virtue of (3.1) and (3.4), the first factor under the integral above is weakly- $\odot$ continuous on $[a, b]$. The proof is complete.

\section{Compactness of the solution operator}

From now on we shall assume that $X$ and $A$ are fixed and such that, for each $(\xi, g) \in X \times B V([a, b] ; X)$, the Cauchy problem (1.2) has a unique $\mathcal{L}^{\infty}$-solution. See Remark 2.2 and Theorem 2.1. Our goal here is to prove the main result of this paper, i.e. a necessary and sufficient condition in order that the family of all $\mathcal{L}^{\infty}$ solutions of the nonhomogeneous linear Cauchy problem (1.2), when $\xi$ ranges in a given subset in $X$ and $g$ ranges in a bounded subset in $B V([a, b] ; X)$, be relatively compact in $L^{p}(a, b ; X)$ for each $p \in[1,+\infty)$. This condition is in fact an extension (in the linear case only - see Theorem 6.1 below) of Theorem 1.1 in VRABIE 28 from mild, or $C^{0}$-solutions to $\mathcal{L}^{\infty}$-solutions, allowing of course the right-hand side in (1.2) to be a measure generated by a function of bounded variation. Let $\xi \in X$ and $g \in B V([a, b] ; X)$, and denote by $u=Q(\xi, g)$ the unique $\mathcal{L}^{\infty}$-solution of the Cauchy problem (1.2) corresponding to $\xi$ and $g$.

Remark 5.1. Since $\{S(t) ; t \geq 0\}$ is a semigroup of contractions, we have

$$
\|u(t)\| \leq\|\xi\|+\operatorname{Var}(g,[a, b]) .
$$


The next simple lemma will prove useful in what follows.

Lemma 5.1. For each $g \in B V([a, b] ; X)$ and $h \in(0, b-a)$ we have

$$
\int_{a}^{b-h}\left\|\int_{t}^{t+h} S(t+h-s) d g(s)\right\| d t \leq h \operatorname{Var}(g,[a, b])
$$

and

$$
\int_{a+h}^{b}\left\|\int_{t-h}^{t} S(t-s) d g(s)\right\| d t \leq h \operatorname{Var}(g,[a, b])
$$

Proof. We shall prove only the first inequality, the second one being obtained via very similar arguments. Since $t<s \leq t+h$ if and only if $s-h \leq t<s$, we have $\chi_{(t, t+h]}(s)=\chi_{[s-h, s)}(t)$ for each $t \in[a, b-h]$ and each $s \in[a+h, b]$. Let us denote $V_{g}(s)=\operatorname{Var}(g,[a, s])$. Since

$$
\int_{t}^{t+h} S(t+h-s) d g(s)=\int_{a}^{b} \chi_{(t, t+h]}(s) S(t+h-s) d g(s)+S(h)[g(t+0)-g(t)],
$$

and $\|g(t+0)-g(t)\|=0$ a.e. for $t \in[a, b]$, we have

$$
\begin{gathered}
\int_{a}^{b-h}\left\|\int_{t}^{t+h} S(t+h-s) d g(s)\right\| d t \\
=\int_{a}^{b-h}\left\|\int_{a}^{b} \chi_{(t, t+h]}(s) S(t+h-s) d g(s)\right\| d t \leq \int_{a}^{b} \int_{a+h}^{b} \chi_{[s-h, s)}(t) d V_{g}(s) d t \\
=\int_{a+h}^{b}\left(\int_{a}^{b} \chi_{[s-h, s)}(t) d t\right) d V_{g}(s) \leq h \operatorname{Var}(g,[a, b]) .
\end{gathered}
$$

The proof is complete.

We recall that a subset $\mathcal{G}$ in $B V([a, b] ; X)$ is of equibounded variation if there exists $m_{\mathcal{G}}>0$ such that

$$
\operatorname{Var}(g,[a, b]) \leq m_{\mathcal{G}}
$$

for each $g \in \mathcal{G}$. For the sake of completeness and simplicity we recall the following specific form of a theorem due to GUTMAN [13], which is the main ingredient in the proof of our main result. Variants of this result may be found in SIMON [21] and VRABIE [27].

Theorem 5.1 (Gutman). A subset $U$ in $L^{p}([a, b] ; X), 1 \leq p<\infty$, is strongly relatively sequentially compact if and only if $U$ is $p$-equi-integrable and, in addition, for each $\varepsilon>0$ there exists a compact subset $C_{\varepsilon}$ in $X$ such that for each $u \in U$ there exists a measurable subset $E_{\varepsilon, u}$ in $[a, b]$ whose Lebesgue measure is less than $\varepsilon$ and such that $u(t) \in C_{\varepsilon}$ for each $u \in U$ and $t \in[a, b] \backslash E_{\varepsilon, u}$.

We may now proceed to the statement of our main result. 
Theorem 5.2. Let $A: D(A) \subseteq X \rightarrow X$ be the infinitesimal generator of a $C_{0}$ semigroup of contractions $\{S(t) ; t \geq 0\}$, let $\mathcal{D}$ be a bounded subset in $X$, and $\mathcal{G}$ a subset in $B V([a, b] ; X)$ of equibounded variation. Then $Q(\mathcal{D}, \mathcal{G})$ is relatively compact in $L^{p}(a, b ; X)$ for each $p \in[1,+\infty)$ if and only if for each $\varepsilon>0$ there exists a relatively compact subset $C_{\varepsilon}$ in $X$ such that, for each $(\xi, g) \in \mathcal{D} \times \mathcal{G}$, there exists a subset $E_{\varepsilon, \xi, g}$ in $[a, b]$ whose Lebesgue measure is less that $\varepsilon$, and such that $Q(\xi, g)(t) \in C_{\varepsilon}$ for each $(\xi, g) \in \mathcal{D} \times \mathcal{G}$ and each $t \in[a, b] \backslash E_{\varepsilon, \xi, g}$.

Proof. By virtue of Theorem 5.1, the necessity is obvious. To prove the sufficiency, we also make use of the same Theorem 5.1. Let us observe first that, by virtue of the Lebesgue Dominated Convergence Theorem, it suffices to show that $Q(\mathcal{D}, \mathcal{G})$ is relatively compact in $L^{1}(a, b ; X)$, and bounded in $\mathcal{L}^{\infty}(a, b ; X)$. To this aim, let us recall that there exist $m_{\mathcal{D}}>0$ and $m_{\mathcal{G}}>0$ such that

$$
\|\xi\| \leq m_{\mathcal{D}} \text { and } \operatorname{Var}(g,[a, b]) \leq m_{\mathcal{G}}
$$

for each $(\xi, g) \in \mathcal{D} \times \mathcal{G}$. Then, by Remark 5.1, we have

$$
\|Q(\xi, g)(t)\| \leq m_{\mathcal{D}}+m_{\mathcal{G}}
$$

for each $(\xi, g) \in \mathcal{D} \times \mathcal{G}$ and $t \in[a, b]$. In order to prove that $Q(\mathcal{D}, \mathcal{G})$ is 1-equiintegrable, let $\varepsilon>0,(\xi, g) \in \mathcal{D} \times \mathcal{G}$, and let $C_{\varepsilon}$ and $E_{\varepsilon, \xi, g}$ be the two subsets having the properties mentioned by hypotheses. A simple computational argument, along with Lemma 5.1. shows that

$$
\begin{gathered}
\int_{a}^{b-h}\|Q(\xi, g)(t+h)-Q(\xi, g)(t)\| d t \leq \int_{a}^{b-h}\|Q(\xi, g)(t+h)-S(h) Q(\xi, g)(t)\| d t \\
+\int_{a}^{b-h}\|S(h) Q(\xi, g)(t)-Q(\xi, g)(t)\| d t \leq \int_{a}^{b-h}\left\|\int_{t}^{t+h} S(t+h-s) d g(s)\right\| d t \\
+\int_{[a, b] \backslash E_{\varepsilon, \xi, g}}\|S(h) Q(\xi, g)(t)-Q(\xi, g)(t)\| d t \\
\quad+\int_{E_{\varepsilon, \xi, g}}\|S(h) Q(\xi, g)(t)-Q(\xi, g)(t)\| d t \\
\leq h \operatorname{Var}(g,[a, b])+\int_{[a, b] \backslash E_{\varepsilon, \xi, g}}\|S(h) Q(\xi, g)(t)-Q(\xi, g)(t)\| d t+2 M_{\mathcal{G}} \varepsilon
\end{gathered}
$$

for each $(\xi, g) \in \mathcal{D} \times \mathcal{G}$ and $h \in(0, b-a]$, where $M_{\mathcal{G}}=m_{\mathcal{D}}+m_{\mathcal{G}}$. As $Q(\xi, g)(t) \in C_{\varepsilon}$ for each $(\xi, g) \in \mathcal{D} \times \mathcal{G}$ and each $t \in[a, b] \backslash E_{\varepsilon, \xi, g}$, while $C_{\varepsilon}$ is relatively compact in $X$, there exists $\delta(\varepsilon) \in(0, b-a]$ such that, for each $h \in(0, \delta(\varepsilon)]$,

$$
\|S(h) Q(\xi, g)(t)-Q(\xi, g)(t)\| \leq \varepsilon
$$

uniformly for $(\xi, g) \in \mathcal{D} \times \mathcal{G}$ and $t \in[a, b] \backslash E_{\varepsilon, \xi, g}$. Then, taking account of (5.1), (5.2) and the preceding inequalities, we obtain

$$
\int_{a}^{b-h}\|Q(\xi, g)(t+h)-Q(\xi, g)(t)\| d t \leq\left(b-a+m_{\mathcal{G}}+2 M_{\mathcal{G}}\right) \varepsilon
$$


for each $(\xi, g) \in \mathcal{D} \times \mathcal{G}$ and $h \in(0, \delta(\varepsilon)] \cap(0, \varepsilon]$. Obviously, this relation shows that $Q(\mathcal{D}, \mathcal{G})$ is 1 -equi-integrable. By Theorem 5.1 it is relatively compact in $L^{1}(a, b ; X)$, and this completes the proof.

Remark 5.2. If $X$ is finite dimensional, then, for each bounded subset $\mathcal{D}$ in $X$ and each subset $\mathcal{G}$ in $B V([a, b] ; X)$ of equibounded variation, $Q(\mathcal{D}, \mathcal{G})$ is relatively compact in $L^{p}(a, b ; X)$ and thus $p$-equi-integrable. This follows from the observation that, by virtue of Remark 5.1, the set $\{Q(\xi, g)(t) ;(\xi, g) \in \mathcal{D} \times \mathcal{G}, t \in[a, b]\}$ is bounded and (inasmuch as $X$ is finite dimensional) also relatively compact. So we are in the hypotheses of Theorem 5.2 and the conclusion follows.

To see that in infinite dimensional Banach spaces the $p$-equi-integrability condition is not an intrinsic property of the set $Q(\mathcal{D}, \mathcal{G})$ with $\mathcal{D}$ and $\mathcal{G}$ bounded, or of equibounded variation, let us analyze the following example, which is an adaptation from VRABIE [26].

Example 5.1. Let $X=L_{2 \pi}^{2}(\mathbb{R})$ be the space of all equivalence classes, with respect to the almost everywhere equality on $\mathbb{R}$, of measurable and $2 \pi$-periodic functions from $\mathbb{R}$ to $\mathbb{R}$. Endowed with the $L^{2}(0,2 \pi ; \mathbb{R})$-norm, this is a real Hilbert space. Let $A: D(A) \subseteq H \rightarrow H$ be defined by $D(A)=\left\{u \in H ; u^{\prime} \in H\right\}$ and $A u=u^{\prime}$ for each $u \in D(A)$. Obviously, $A$ generates a $C_{0}$-group of isometries on $H$, i.e. the translation group. Let $\mathcal{D}=\{0\}$ and $\mathcal{G}=\left\{t \mapsto-\frac{1}{n} \cos \{n(t+\cdot)\} ; n \in \mathbb{N}^{*}\right\}$. It is easy to see that $\mathcal{G}$ is of equibounded variation on $[0,1]$. On the other hand, in this case, $Q(\mathcal{D}, \mathcal{G})=\left\{t \mapsto \sin \{n(t+\cdot)\} ; n \in \mathbb{N}^{*}\right\}$, which is not $L^{p}$-equicontinuous on $[0,1]$ because the family is not relatively compact in $L^{p}\left(0,1 ; L^{2}(0,2 \pi ; \mathbb{R})\right)$.

\section{Some COnSEQuences}

In this section we include some useful consequences of Theorem 5.2. We begin with a specific linear version of a compactness result due to the author. See VRABIE 28]. Here and hereafter, $M$ denotes the solution operator which assigns to each $(\xi, f)$ in $X \times L^{1}(a, b ; X)$ the unique mild, or $C^{0}$-solution of the problem (1.1) corresponding to $(\xi, f)$.

Theorem 6.1 (Vrabie). Let $A: D(A) \subseteq X \rightarrow X$ be the infinitesimal generator of a $C_{0}$-semigroup of contractions, let $\mathcal{D}$ be a bounded subset in $X$, and $\mathcal{F}$ a bounded subset in $L^{1}([a, b] ; X) \times \overline{D(A)}$. Then, $M(\mathcal{D}, \mathcal{F}):=\{M(\xi, f),(\xi, f) \in \mathcal{D} \times \mathcal{F}\}$ is relatively compact in $L^{p}(a, b ; X)$, for each $p \in[1,+\infty)$, if and only if for each $\varepsilon>0$ there exists a relatively compact subset $C_{\varepsilon}$ in $X$ such that, for each $(\xi, f) \in \mathcal{D} \times \mathcal{F}$, there exists a subset $E_{\varepsilon, \xi, f}$ in $[a, b]$ whose Lebesgue measure is less than $\varepsilon$, and such that $M(\xi, f)(t) \in C_{\varepsilon}$ for each $(\xi, f) \in \mathcal{D} \times \mathcal{F}$ and $t \in[a, b] \backslash E_{\varepsilon, \xi, f}$.

Proof. The conclusion follows from Remark 2.6 and Theorem 5.2

Corollary 6.1. Let $A: D(A) \subseteq X \rightarrow X$ be the infinitesimal generator of a $C_{0^{-}}$ semigroup of contractions $\{S(t) ; t \geq 0\}$, let $\mathcal{D}$ be a bounded subset in $X$, and $\mathcal{G}$ a subset in $B V([a, b] ; X)$ of equibounded variation. If

$$
\{Q(\xi, g)(t) ;(\xi, g) \in \mathcal{D} \times \mathcal{G}, t \in[a, b]\}
$$

is relatively compact in $X$, then $Q(\mathcal{D}, \mathcal{G})$ is relatively compact in $L^{p}(a, b ; X)$, for each $p \in[1,+\infty)$. 
The next lemma, closely related to the well-known Egoroff's Theorem (see DuNFORD, SchWARTz [10], Theorem 12, p. 149), will play a central role in the proof of the next theorem.

Lemma 6.1. Let $U$ be a nonempty and bounded subset in $L^{1}(a, b ; X)$, and let $\left\{Q_{h} ; h \in(0, b-a]\right\}$ be a family of (possible nonlinear) operators from $U$ to $L^{1}(a, b ; X)$ such that $\lim _{h \downarrow 0} \int_{a}^{b}\left\|Q_{h} u(t)\right\| d t=0$, uniformly for $u \in U$. Then, for each $\varepsilon>0$, there exists a sequence $\left(h_{n}\right)_{n}$, decreasing to 0 , with the property that, for each $u \in U$, there exists a subset $E_{\varepsilon, u}$ in $[a, b]$ whose Lebesgue measure $\lambda\left(E_{\varepsilon, u}\right)$ is less than $\varepsilon$ and $\lim _{n} Q_{h_{n}} u(t)=0$, uniformly for $u \in U$ and $t \in[a, b] \backslash E_{\varepsilon, u}$.

Proof. Let $\varepsilon>0$, and let us choose two sequences $\left(h_{n}\right)_{n}$ and $\left(a_{n}\right)_{n}$, both decreasing to 0 , such that

$$
\sum_{n=0}^{\infty} a_{n} \leq \varepsilon \text { and } \int_{a}^{b}\left\|Q_{h_{n}} u(t)\right\| d t<a_{n}^{2}
$$

for each $n \in \mathbb{N}$ and $u \in U$. For $u \in U$ and $n \in \mathbb{N}$, let us define

$$
E_{u}^{n}=\left\{t \in[a, b] ;\left\|Q_{h_{n}} u(t)\right\| \geq a_{n}\right\} \quad \text { and } \quad E_{\varepsilon, u}=\bigcup_{n \in \mathbb{N}} E_{u}^{n} .
$$

In view of (6.1), one may easily verify that $\lambda\left(E_{u}^{n}\right)<a_{n}$ for each $n \in \mathbb{N}$, and thus $\lambda\left(E_{\varepsilon, u}\right)<\varepsilon$. From the definition of $E_{\varepsilon, u}$, we deduce that $\left\|Q_{h_{n}} u(t)\right\|<a_{n}$ for each $n \in \mathbb{N}, u \in U$, and $t \in[a, b] \backslash E_{\varepsilon, u}$, and this completes the proof.

Theorem 6.2. If $A: D(A) \subseteq X \rightarrow X$ is the infinitesimal generator of a compact $C_{0}$-semigroup of contractions, $\mathcal{D}$ is a bounded subset in $X$, and $\mathcal{G}$ is a subset in $B V([a, b] ; X)$ of equibounded variation, then, for each $p \in[1,+\infty)$, the set

$$
Q(\mathcal{D}, \mathcal{G}):=\{Q(\xi, g) ;(\xi, g) \in \mathcal{D} \times \mathcal{G}\}
$$

is relatively compact in $L^{p}(a, b ; X)$.

Proof. We shall prove that $Q(\mathcal{D}, \mathcal{G})$ satisfies the hypotheses of Theorem 5.2. To this aim, let us observe that, by virtue of Lemma 5.1 for $u=Q(\xi, g)$ we have

$$
\int_{a+h}^{b}\|u(t)-S(h) u(t-h)\| d t \leq \int_{a+h}^{b}\left\|\int_{t-h}^{t} S(t-s) d g(s)\right\| d t \leq h \operatorname{Var}(g,[a, b]),
$$

for each $(\xi, g) \in \mathcal{D} \times \mathcal{G}$ and $h \in(0, b-a]$. Inasmuch as $\mathcal{G}$ is of equibounded variation, we get

$$
\lim _{h \downarrow 0} \int_{a+h}^{b}\|u(t)-S(h) u(t-h)\| d t=0,
$$

uniformly for $(\xi, g) \in \mathcal{D} \times \mathcal{G}$

Let us define $U=Q(\mathcal{D}, \mathcal{G})$ and $Q_{h}: U \rightarrow L^{1}(a, b ; X)$ by

$$
\left(Q_{h} u\right)(t)= \begin{cases}0 & \text { if } t \in[a, a+h] \\ u(t)-S(h) u(t-h) & \text { if } t \in(a+h, b] .\end{cases}
$$

From (6.2) it follows that Lemma 6.1 applies, and therefore, for each $\varepsilon>0$, there exists $E_{\varepsilon, u} \subseteq[a, b]$ with $\lambda\left(E_{\varepsilon, u}\right)<\varepsilon$, and $\left(h_{n}\right)_{n}$ which decreases to 0 , such that

$$
\lim _{n}\left\|u(t)-S\left(h_{n}\right) u\left(t-h_{n}\right)\right\|=0,
$$


uniformly for $u \in Q(\mathcal{D}, \mathcal{G})$ and $t \in[a, b] \backslash E_{\varepsilon, u}$. Since for each $n \in \mathbb{N}$ the contraction $S\left(h_{n}\right)$ is compact, and $\{u(s) ; u \in Q(\mathcal{D}, \mathcal{G}), s \in[a, b]\}$ is bounded in $X$, it follows that the set

$$
C_{\varepsilon}=\left\{u(t) ; u \in Q(\mathcal{D}, \mathcal{G}), t \in[a, b] \backslash E_{\varepsilon, u}\right\}
$$

is relatively compact in $X$. Consequently, we are in the hypotheses of Theorem 5.2 Hence $Q(\mathcal{D}, \mathcal{G})$ is relatively compact in $L^{p}(a, b ; X)$ for each $p \in[1,+\infty)$, and this completes the proof.

Theorem 6.3 (Baras, Hassan, Veron). If $A: D(A) \subseteq X \rightarrow X$ is the infinitesimal generator of a compact $C_{0}$-semigroup of contractions, $\mathcal{D}$ is a bounded subset in $X$, and $\mathcal{F}$ is a bounded subset in $L^{1}(a, b ; X)$, then, for each $p \in[1,+\infty)$, the set

$$
M(\mathcal{D}, \mathcal{F}):=\{M(\xi, f) ;(\xi, f) \in \mathcal{D} \times \mathcal{F}\}
$$

is relatively compact in $L^{p}(a, b ; X)$.

Proof. The conclusion follows from Remark 2.6 and Theorem 6.2

\section{Evolution equations With "SPATial" MEASURES AS DATA}

Let $X$ be a real Banach space, and let us consider the Cauchy problem (1.2), where $A: D(A) \subseteq X \rightarrow X$ generates a compact $C_{0}$-semigroup of contractions, $\xi \in X^{c}$, and $g \in B V\left([a, b] ; X^{c}\right)$, where $X^{c}$ is the sequential completion of $X$ in $\sigma\left(X, X^{\odot}\right)$, i.e. in the so-called weak- $\odot$ topology. See Section 2. By the HahnBanach Theorem (see Hille, Phillips [14], Theorem 2.1.2, p. 29) it readily follows that $X^{c}$ is a closed subspace of $X^{* *}$. We notice that whenever $X$ is reflexive, and thus $X^{\odot}=X^{*}$, we have $X^{c}=X$, and therefore the problem (1.2) can be easily treated by the previously developed theory. This is no longer true in the nonreflexive case when $X^{c} \neq X$, and this explains why, throughout this section, we constantly assume that $X$ is nonreflexive, although all the abstract results hold (trivially) true in general. Another reason, much more subtle, for doing this, is that the analysis of partial differential equations involving measures with respect to the spatial argument relies heavily on nonreflexive settings and techniques, such as $L^{1}$ spaces and vague topologies. See Example 7.1 below. We begin with the following auxiliary result.

Lemma 7.1. Let $A: D(A) \subseteq X \rightarrow X$ be the infinitesimal generator of a $C_{0}$ semigroup of contractions, and let $\left(g_{k}\right)_{k}$ be a sequence in $B V([a, b] ; X)$ with equibounded variation, and such that

$$
\lim _{k} g_{k}(t)=0
$$

for each $t \in[a, b]$ in $\sigma\left(X, X^{\odot}\right)$. Then, for each $t \in[a, b]$, we have

$$
\lim _{k} \int_{a}^{t} S(t-s) d g_{k}(s)=0
$$

in $\sigma\left(X, X^{\odot}\right)$.

Proof. Let $x^{\odot} \in X^{\odot}$. Then, for each $k \in \mathbb{N}$, the function $f_{k}:[a, b] \rightarrow \mathbb{R}$, defined by $f_{k}(t)=\left(g_{k}(t), x^{\odot}\right)$, belongs to $B V([a, b] ; \mathbb{R})$, and $\lim _{k} f_{k}(t)=0$ for each $t \in[a, b]$. In addition, the sequence $\left(f_{k}\right)_{k}$ is of equibounded variation. So, by the classical 
Helly-Bray Theorem (see GRAVEs [12, Theorem 2.3, p. 283), it follows that, for each $\varphi_{i} \in C([a, t] ; \mathbb{R})$ and $x_{i}^{\odot} \in X^{\odot}, i=1,2, \ldots, n$, we have

$$
\lim _{k} \int_{a}^{t}\left(d g_{k}(s) \sum_{i=1}^{n} \varphi_{i}(s) x_{i}^{\odot}\right)=0 .
$$

Finally, let us observe that the set of all functions of the form $s \mapsto \sum_{i=1}^{n} \varphi_{i}(s) x_{i}^{\odot}$ is dense in $C\left([a, t] ; X^{\odot}\right)$ with respect to the sup-norm, and this completes the proof.

In order to give a precise meaning to (1.2) in this more general setting, we need the following convergence result.

Theorem 7.1. Let $A: D(A) \subseteq X \rightarrow X$ be the infinitesimal generator of a compact $C_{0}$-semigroup of contractions, let $\xi \in X^{c}, g \in B V\left([a, b] ; X^{c}\right)$, and let $\left(\xi_{k}\right)_{k}$ and $\left(g_{k}\right)_{k}$ be two sequences, in $X$ and in $B V([a, b] ; X)$ respectively, such that $\left(g_{k}\right)_{k}$ has equibounded variation, and

$$
\begin{cases}\lim _{k \rightarrow \infty} \xi_{k}=\xi & \text { in } \sigma\left(X, X^{\odot}\right), \\ \lim _{k \rightarrow \infty} g_{k}(t)=g(t) & \text { for each } t \in[a, b] \text { in } \sigma\left(X, X^{\odot}\right) .\end{cases}
$$

Then, there exists $u \in \mathcal{L}^{\infty}\left(a, b ; X^{c}\right)$ with $u(t) \in X$ a.e. for $t \in[a, b]$, and such that, for each $p \in[1, \infty)$,

$$
\lim _{k \rightarrow \infty} Q\left(\xi_{k}, g_{k}\right)=u
$$

strongly in $L^{p}(a, b ; X)$, and pointwise in $\sigma\left(X, X^{\odot}\right)$. In addition, for each $x^{\odot} \in X^{\odot}$, we have

$$
\begin{gathered}
\left(u(t), x^{\odot}\right)=\left(\xi, S(t-a)^{\odot} x^{\odot}\right)+\int_{a}^{t}\left(d g(s), S(t-s)^{\odot} x^{\odot}\right), \\
u(t+0)-u(t)=g(t+0)-g(t) \text { and } u(s)-u(s-0)=g(s)-g(s-0),
\end{gathered}
$$

where the one-sided limits on the left-hand sides of (7.3) are considered in the weak$\odot$ topology on $X$.

Proof. Observe that, for each $k, p \in \mathbb{N}$, each $t \in[a, b]$, and each $x^{\odot} \in X^{\odot}$, we have

$$
\begin{gathered}
\left(Q\left(\xi_{k}, g_{k}\right)(t)-Q\left(\xi, g_{p}\right)(t), x^{\odot}\right) \\
=\left(\xi_{k}-\xi_{p}, S(t-a)^{\odot} x^{\odot}\right)+\left(\int_{a}^{t} S(t-s) d\left(g_{k}-g_{p}\right)(s), x^{\odot}\right) .
\end{gathered}
$$

where $\left\{S^{\odot}(t) ; t \geq 0\right\}$ is the sun dual semigroup. See Section 2. By virtue of Lemma 7.1 we deduce that $\lim _{k, p}\left(\int_{a}^{t} S(t-s)\left(d\left(g_{k}-g_{p}\right)(s), x^{\odot}\right)=0\right.$ for each $t \in[a, b]$ and $x^{\odot} \in X^{\odot}$. Furthermore, we have $\lim _{k, p}\left(\xi_{k}-\xi_{p}, S(t-a)^{\odot} x^{\odot}\right)=$ 0 for each $t \in[a, b]$ and $x^{\odot} \in X^{\odot}$. Summing up, we conclude that, for each $t \in[a, b],\left(Q\left(\xi_{k}, g_{k}\right)(t)\right)_{k}$ is a Cauchy sequence in the weak- $\odot$ topology. Therefore there exists $u:[a, b] \rightarrow X^{c}$ such that $\lim _{k} Q\left(\xi_{k}, g_{k}\right)(t)=u(t)$ weakly- $\odot$ in $X^{c}$. Fix $p \in[1,+\infty)$, and observe that, by virtue of Theorem 5.2 , on a subsequence at least, $\lim _{k} Q\left(\xi_{k}, g_{k}\right)=v$ strongly in $L^{p}(a, b ; X)$. So $v$ must coincide with $u$ a.e. on $[a, b]$. As $\left\{Q\left(\xi_{k}, g_{k}\right) ; k \in \mathbb{N}\right\}$ is relatively compact in $L^{p}(a, b ; X)$, it follows that $\left(Q\left(\xi_{k}, g_{k}\right)\right)_{k}$ itself converges in $L^{p}(a, b ; X)$ to $u$, and this proves (17.1). Finally, let 
us observe that (7.2) is a direct consequence of Lemma 7.1, while (7.3) follows by using the very same arguments as in Theorem 3.1 , and this achieves the proof.

Remark 7.1. By Lemma 7.1, one may easily verify that the limit in (17.1) does not depend on the choice of the sequences $\left(\xi_{k}\right)_{k}$ and $\left(g_{k}\right)_{k}$ which approximate $\xi$ and $g$. Therefore, Theorem 7.1 allows us to extend the concept of the $\mathcal{L}^{\infty}$-solution to the case in which $\xi \in X^{c}$ and $g \in B V\left([a, b] ; X^{c}\right)$, whenever the latter can be approximated in the pointwise convergence weak- $\odot$ topology by a sequence of $X$ valued functions $\left(g_{k}\right)_{k}$ with equibounded variation and, of course, the semigroup generated by $A$ is compact. More precisely, we have

Definition 7.1. Let $\xi \in X^{c}$ and $g \in B V\left([a, b] ; X^{c}\right)$. A function $u \in \mathcal{L}^{\infty}\left(a, b ; X^{c}\right)$ with $u(t) \in X$ a.e. for $t \in[a, b]$, and satisfying (7.2), is called an $\mathcal{L}^{\infty}$-generalized solution of the problem (1.2) on $[a, b]$.

We also notice that under these circumstances, the operator $A$ has a smoothing effect on the data in the sense that, for each $\xi \in X^{c}$ and $g \in B V\left([a, b] ; X^{c}\right)$ as in Theorem [7.1, the $\mathcal{L}^{\infty}$-generalized solution $u$ is an $X$-valued function and not an $X^{c}$-valued one, as we might expect at first glance.

Since $X^{c}$ obviously depends on $A$, in all that follows, we call it the space of admissible measures for $A$. A prototype of the situation described in Theorem 7.1 is illustrated by the following suggestive example.

Example 7.1. Let $\Omega$ be a bounded domain in $\mathbb{R}^{n}$ with sufficiently smooth boundary $\Gamma$, and let us consider the sequence of linear parabolic problems

$$
\begin{cases}\frac{\partial u_{k}}{\partial t}=\Delta u_{k}+f_{k} & \text { on }(0, T) \times \Omega, \\ u_{k}=0 & \text { in }(0, T) \times \Gamma, \\ u_{k}(0)=\xi_{k}, & \end{cases}
$$

where $\left(\xi_{k}\right)_{k}$ and $\left(f_{k}\right)_{k}$ are two bounded sequences, in $L^{1}(\Omega)$ and $\left.L^{1}((0, T) \times \Omega)\right)$ respectively. Let $C_{0}(\bar{\Omega})$ be the space of continuous functions from $\bar{\Omega}$ to $\mathbb{R}$ vanishing on $\Gamma$, let $\left(L^{1}(\Omega)\right)^{c}$ be the sequential completion of $L^{1}(\Omega)$ in $\sigma\left(L^{1}(\Omega), C_{0}(\bar{\Omega})\right)$, and assume that there exist $\xi \in\left(L^{1}(\Omega)\right)^{c}$ and $g \in B V\left([0, T] ;\left(L^{1}(\Omega)\right)^{c}\right)$ such that

$$
\lim _{k} \int_{\Omega} \int_{0}^{t} f_{k}(s, x) \varphi(x) d s d x=g(t) \text { and } \lim _{k} \int_{\Omega} \xi_{k}(x) \varphi(x) d x=\xi
$$

for each $\varphi \in C_{0}(\bar{\Omega})$. The problem is to give meaning to the limiting equation. A natural way to approach (7.4) can be described as follows. Take $X=L^{1}(\Omega)$, and let $A: D(A) \subseteq X \rightarrow X$ be defined by

$$
D(A)=\left\{u \in W_{0}^{1,1}(\Omega) ; \Delta u \in L^{1}(\Omega)\right\} \text { and } A u=\Delta u
$$

for each $u \in D(A)$. It is well-known that $A$ generates a compact $C_{0}$-semigroup of contractions $\{S(t) ; t \geq 0\}$ on $X$. See Baras, Hassan, Veron [2]. Let $\left\{S(t)^{\odot} ; t \geq 0\right\}$ be the sun dual semigroup on $X^{\odot}$. In our case, one may easily verify that $X^{\odot}=C_{0}(\bar{\Omega})$. At this point, let us observe that Theorem [7.1]applies, and thus $\lim _{k} u_{k}=u$ exists strongly in $L^{p}\left(0, T L^{1}(\Omega)\right)$ for each $p \in[1, \infty)$, and pointwise in $\sigma\left(L^{1}(\Omega),\left(L^{1}(\Omega)\right)^{c}\right)$. So, $u$ can be interpreted as a generalized solution 
of the limiting problem

$$
\begin{cases}d u=(\Delta u) d t+d g & \text { in }(0, T) \times \Omega \\ u=0 & \text { on }(0, T) \times \Sigma \\ u(0)=\xi & \end{cases}
$$

We notice that the space $\left(L^{1}(\Omega)\right)^{c}$ contains all the Dirac measures concentrated in $\Omega$. Thus, in the problem above, we are allowed to consider forcing terms of the form $d g=\sum_{i, j=1}^{m} a_{i j} \delta\left(t-t_{i}\right) \otimes \delta\left(x-x_{j}\right)$ and $\xi=\sum_{j=1}^{m} b_{j} \delta\left(x-y_{j}\right)$, with $a_{i, j}, b_{j} \in \mathbb{R}$, $t_{i} \in[0, T]$ and $x_{j}, y_{j} \in \Omega$, for $i, j=1,2, \ldots, m$.

\section{The semilinear evolution equation $d u=\{A u+f(t, u)\} d t+d g$}

Let $X$ be a real Banach space. Let $\mathbb{I}$ be a nonempty and open interval in $\mathbb{R}$ and $U$ a nonempty and open subset in $X$. We denote by $B V(\mathbb{I} ; X)$ the space of all functions from $\mathbb{I}$ to $X$ whose restrictions to any interval $[a, b] \subset \mathbb{I}$ are in $B V([a, b] ; X)$. Let $A: D(A) \subseteq X \rightarrow X$ be the infinitesimal generator of a $C_{0^{-}}$ semigroup of contractions $\{S(t) ; t \geq 0\}, f: \mathbb{I} \times U \rightarrow X$ a continuous function, $g \in B V(\mathbb{I} ; X), a \in \mathbb{I}$, and $\xi \in X$. Consider the Cauchy problem

$$
\left\{\begin{array}{l}
d u=\{A u+f(t, u)\} d t+d g \\
u(a)=\xi
\end{array}\right.
$$

An $\mathcal{L}^{\infty}$-solution of the problem (8.1) on $[a, c]$ is a function $u:[a, c] \rightarrow U$ which satisfies $u \in \mathcal{L}^{\infty}(a, c ; X)$ and

$$
u(t)=S(t-a) \xi+\int_{a}^{t} S(t-s) f(s, u(s)) d s+\int_{a}^{t} S(t-s) d g(s)
$$

for each $t \in[a, c]$.

The next abstract local existence theorem, extending a well-known result due to PAZY [19], proves useful in the study of semilinear parabolic problems with distributed measures, as we shall see in the next section. Pazy's main result in [19] refers to the special case $g \equiv 0$.

Theorem 8.1. If $A: D(A) \subseteq X \rightarrow X$ is the infinitesimal generator of a compact $C_{0}$-semigroup of contractions $\{S(t) ; t \geq 0\}$, and $f: \mathbb{I} \times U \rightarrow X$ is continuous, then, for each $a \in \mathbb{I}$ and each $\xi \in U$ with $g(a+0)-g(a)+\xi \in U$, there exists $b>a$ with $[a, b] \subseteq \mathbb{I}$, and such that the problem (8.1) has at least one $\mathcal{L}^{\infty}$-solution defined on $[a, b]$.

First, we shall prove

Lemma 8.1. If $A: D(A) \subseteq X \rightarrow X$ is the infinitesimal generator of a compact $C_{0}$-semigroup of contractions $\{S(t) ; t \geq 0\}$, and $f: \mathbb{I} \times X \rightarrow X$ is continuous and bounded, then for each $[a, c] \subset \mathbb{I}$, and each $\xi \in X$, the problem (8.1) has at least one $\mathcal{L}^{\infty}$-solution defined on $[a, c]$.

Proof. Let $[a, c] \subset \mathbb{I}, \xi \in X, \lambda>0$, and consider the delay equation

$$
\left\{\begin{array}{l}
d u_{\lambda}=\left\{A u_{\lambda}+f\left(t, u_{\lambda}(\cdot-\lambda)\right)\right\} d t+d g \\
u_{\lambda}(s)=\xi, \quad s \in[a-\lambda, a] .
\end{array}\right.
$$

Since the semigroup is continuous in the uniform operator topology from $(0,+\infty)$ to $\mathcal{L}(X)$, being compact, see PAZY [20], Theorem 3.3, p. 48, and $f$ is continuous too, by virtue of Theorem [2.1, it easy to see that (8.3) has a unique $\mathcal{L}^{\infty}$-solution defined successively on $[a, a+\lambda],[a+\lambda, a+2 \lambda]$ and so on. For each $n \in \mathbb{N}^{*}$ let us 
denote by $u_{n}$ the unique solution of the problem (8.3) corresponding to $\lambda=1 / n$. As $f$ is bounded, it follows that the family

$$
\mathcal{G}=\left\{t \mapsto \int_{a}^{t} f\left(s, u_{n}(s-1 / n)\right) d s+g(t) ; n \in \mathbb{N}^{*}\right\}
$$

is of equibounded variation. From Theorem 6.2 we deduce that, for each $p \in[1, \infty)$, the set $\left\{u_{n} ; n \in \mathbb{N}^{*}\right\}$ is relatively compact in $L^{p}(a, c ; X)$. So, we may assume with no loss of generality that $\lim _{n} u_{n}=u$ exists in $L^{1}(a, c ; X)$. On the other hand, we also have $\lim _{n} u_{n}(s-1 / n)=u(s)$ a.e. for $s \in[a, c]$. Since $f$ is continuous and bounded, by the Lebesgue Dominated Convergence Theorem, we deduce that

$$
\lim _{n} \int_{a}^{t} f\left(s, u_{n}(s-1 / n)\right) d s=\int_{a}^{t} f(s, u(s)) d s
$$

for each $t \in[a, c]$. Accordingly, passing to the limit for $n$ tending to $\infty$ in the equality

$$
u_{n}(t)=S(t-a) \xi+\int_{a}^{t} S(t-s) f\left(s, u_{n}(s-1 / n)\right) d s+\int_{a}^{t} S(t-s) d g(s),
$$

we conclude that $u$ satisfies (8.2), and thus it is an $\mathcal{L}^{\infty}$-solution of the problem (8.1) on $[a, c]$. The proof is complete.

We now prove Theorem 8.1 .

Proof. Let $a \in \mathbb{I}$ and $\xi \in X$, and denote $\eta=g(a+0)-g(a)+\xi$. Inasmuch as $\mathbb{I}$ and $U$ are open, and $f$ is continuous, there exist $c>a, r>0$, and $M>0$ such that $[a, c] \subset \mathbb{I}, B(\eta, r) \subseteq U$, and

$$
\|f(t, u)\| \leq M
$$

for each $(t, u) \in[a, c] \times B(\eta, r)$. Let let us define $\rho: X \rightarrow X$ by

$$
\rho(y)=\left\{\begin{array}{cl}
y & \text { for } y \in B(\eta, r), \\
\frac{r}{\|y-\eta\|}(y-\eta)+\eta & \text { for } y \in X \backslash B(\eta, r) .
\end{array}\right.
$$

Clearly, $\rho$ maps $X$ to $B(\eta, r)$, and is continuous. Let us define $f_{r}: \mathbb{I} \times X \rightarrow X$ by

$$
f_{r}(t, u)=\left\{\begin{array}{lll}
f(a, \rho(u)) & \text { if } t \in \mathbb{I} \cap(-\infty, a] & \text { and } u \in X, \\
f(t, \rho(u)) & \text { if } t \in[a, c] & \text { and } u \in X, \\
f(c, \rho(u)) & \text { if } t \in \mathbb{I} \cap[c,+\infty) & \text { and } u \in X .
\end{array}\right.
$$

From (8.4), we conclude that $f_{r}$ is bounded. Moreover, since both $f$ and $\rho$ are continuous, it follows that $f_{r}$ is continuous. From Lemma 8.1 we know that the Cauchy problem

$$
\left\{\begin{array}{l}
d u=\left\{A u+f_{r}(t, u)\right\} d t+d g \\
x(a)=\xi
\end{array}\right.
$$

has at least one $\mathcal{L}^{\infty}$-solution, $u:[a, c] \rightarrow X$. Since, $u(a)=\xi$, by (3.1) in Theorem 3.1, there exists $b \in(a, c]$ such that, for each $t \in(a, b]$, we have $u(t) \in B(\eta, r)$. But in this case $\rho(u(t))=u(t)$ for each $t \in[a, b]$, and consequently $f_{r}(s, u(s))$ must coincide with $f(s, u(s))$ for each $s \in[a, b]$. Hence $u:[a, b] \rightarrow X$ is an $\mathcal{L}^{\infty}$-solution of the problem (8.1), and this completes the proof.

We conclude this section with a remarkable consequence of Theorem 8.1 . 
Theorem 8.2. Let $A: D(A) \subseteq X \rightarrow X$ be the infinitesimal generator of a compact $C_{0}$-semigroup of contractions $\{S(t) ; t \geq 0\}$, and $f: \mathbb{I} \times X \rightarrow X$ a continuous function for which there exist $\alpha, \beta \in \mathcal{L}_{\text {loc }}^{1}(\mathbb{I})$ satisfying

$$
\|f(t, u)\| \leq \alpha(t)\|u\|+\beta(t)
$$

a.e. for $t \in \mathbb{I}$, and for each $u \in X$. Then, for each $a, b \in \mathbb{I}$ with $a<b$, each $\xi \in X^{c}$, and each $g \in B V\left([a, b] ; X^{c}\right)$ which can be pointwise approximated in the $\sigma\left(X^{c}, X^{\odot}\right)$ topology by a sequence of functions $\left(g_{k}\right)_{k}$ in $B V([a, b] ; X)$ with equibounded variation, there exists at least one $\mathcal{L}^{\infty}$-generalized solution of (8.1) defined on $[a, b]$.

Proof. Let $\left(\xi_{k}\right)_{k}$ be a sequence in $X$ which converges in the weak- $\odot$ topology to $\xi$, and $\left(u_{k}\right)_{k}$ the sequence of noncontinuable solutions of (8.1) corresponding to $\left(\xi_{k}\right)_{k}$ and $\left(g_{k}\right)_{k}$. First, let us observe that $u_{k}$ is defined at least on $[a, b]$. Indeed, let us assume by contradiction that, for some $k \in \mathbb{N}, u_{k}$ is defined on $[a, c)$ with $c \in(a, b)$. By (8.5), and Lemma 5 in Ahmed [1], which generalizes the wellknown Gronwall's Inequality, it readily follows that $u_{k}$ is bounded on $[a, c)$. Using arguments similar to those in the proof of (3.3), we conclude that there exists $u(c-0) \in X$. Therefore, by Theorem 7.1 the problem (8.1) with the initial data $u(c)=u(c-0)+g(c)-g(c-0)$ has at least one $\mathcal{L}^{\infty}$-solution defined on $[c, c+\delta]$ with $\delta>0$. In view of Proposition 2.1 $u_{k}$ can be continued to the whole interval $[a, c+\delta]$, thereby contradicting the fact that $u_{k}$ is noncontinuable. So, for each $b \in \mathbb{I}, b>a$ and $k \in \mathbb{N}, u_{k}$ is defined at least on $[a, b]$. By the Uniform Boundedness Principle it follows that $\left(\xi_{k}\right)_{k}$ is bounded in $X$, while, by hypotheses, $\left(g_{k}\right)_{k}$ is of equibounded variation on $[a, b]$. Again by (8.5) and Lemma 5 in Ahmed [1, we conclude that $\left(u_{k}\right)_{k}$ is uniformly bounded on $[a, b]$, and therefore the family

of functions $\left\{t \mapsto \int_{a}^{t} f\left(s, u_{k}(s)\right) d s+g_{k}(t) ; k \in \mathbb{N}\right\}$ is of equibounded variation on $[a, b]$. By Theorem 6.2, we conclude that, on a subsequence at least, $\left(u_{k}\right)_{k}$ converges in $L^{p}(a, b ; X)$, for each $p \in[1, \infty)$, to a certain function $u:[a, b] \rightarrow X$. Since $f$ is continuous and we may assume without loss of generality that $\left(u_{k}\right)_{k}$ converges a.e. on $[a, b]$ to $u$, a simple computational argument shows that $u$ is an $\mathcal{L}^{\infty}$-generalized solution of (8.1). This completes the proof.

\section{Some examples}

Let $\Omega$ be a bounded domain in $\mathbb{R}^{n}$ whose boundary $\Gamma$ is of class $C^{\infty}$ and such that $\Omega$ is locally on one side of $\Gamma$. In this section we consider the semi-dynamic elliptic problem

$$
\begin{cases}-\Delta u=0 & \text { in } Q_{T}, \\ d u+\left\{u_{\nu}+\beta(t, u)\right\} d t=d \varphi & \text { on } \Sigma_{T} \\ u(0)=u_{0} & \text { on } \Gamma\end{cases}
$$

as well as the parabolic problem with dynamic boundary conditions

$$
\begin{cases}d u+\{-\Delta u+\alpha(t, u)\} d t=d \eta & \text { in } Q_{T} \\ d u+\left\{u_{\nu}+\beta(t, u)\right\} d t=d \psi & \text { on } \Sigma_{T} \\ u(0)=u_{0}^{\Omega} & \text { in } \Omega \\ u(0)=u_{0}^{\Gamma} & \text { on } \Gamma\end{cases}
$$

where $Q_{T}=(0, T) \times \Omega, \Sigma_{T}=(0, T) \times \Gamma, \alpha, \beta:[0, T] \times \mathbb{R} \rightarrow \mathbb{R}$ are continuous (but not necessarily monotone with respect to $u \in \mathbb{R}$ ), $u_{0}, u_{0}^{\Gamma} \in L^{2}(\Gamma), u_{0}^{\Omega} \in$ 
$L^{2}(\Omega), u_{\nu}$ is the conormal derivative of $u$ at points of $\Gamma, \varphi, \psi \in B V\left([0, T] ; L^{2}(\Gamma)\right)$, and $\eta \in B V\left([0, T] ; L^{2}(\Omega)\right)$. Such problems arise, for instance, from the study of heat transfer in a body $\Omega$ surrounded by a moving fluid. For more details on the significance of (9.1) and (9.2) see BEJEnARU, DíAz, VRABIE [5], and the references therein. We will show next how (9.1) and (9.2) can be analyzed by means of Theorem 8.1. We start with (9.1). Namely, we have

Theorem 9.1. Assume that $\beta:[0, T] \times \mathbb{R} \rightarrow \mathbb{R}$ is continuous, has sublinear growth, and $\varphi \in B V\left([0, T] ; L^{2}(\Gamma)\right)$. Then, for each $u_{0} \in L^{2}(\Gamma)$, there exists $T_{0} \in(0, T)$ such that the problem (9.1) has at least one solution $u:\left[0, T_{0}\right] \rightarrow L^{2}(\Omega)$ satisfying:

(i) for each $t \in\left[0, T_{0}\right)$ and each $s \in\left(0, T_{0}\right]$, we have

$$
\left\{\begin{array}{l}
u_{\mid \Gamma}(t+0)-u_{\mid \Gamma}(t)=\varphi(t+0)-\varphi(t) \\
u_{\mid \Gamma}(s)-u_{\mid \Gamma}(s-0)=\varphi(s)-\varphi(s-0)
\end{array}\right.
$$

(ii) for each interval $\mathbb{I}$ of absolute continuity of $\varphi$ for which $\varphi^{\prime} \in L^{2}\left(\mathbb{I} ; L^{2}(\Gamma)\right)$, we have $u_{\mid \Gamma} \in C\left(\mathbb{I} ; H^{1 / 2}(\Gamma)\right) \cap W^{1,2}\left(\mathbb{I} ; L^{2}(\Gamma)\right), u \in C\left(\mathbb{I} ; H^{1}(\Omega)\right)$, and $u$ satisfies (9.1) a.e. on $\mathbb{I}$.

Proof. Take $H=L^{2}(\Gamma)$, and let us define the operator $A: D(A) \subseteq H \rightarrow H$ by

$$
D(A)=\left\{y \in H^{1 / 2}(\Gamma) ; u_{\nu} \in L^{2}(\Gamma)\right\},
$$

where $u \in H^{1}(\Omega)$ is the unique solution of the elliptic problem

$$
\begin{cases}-\Delta u=0 & \text { in } \Omega \\ u=y & \text { on } \Gamma\end{cases}
$$

and $A y=-u_{\nu}$ for each $u \in D(A)$. It is known that $A$ is a densely defined, self-adjoint operator which generates a compact analytic semigroup of contractions on $H$. See Bejenaru, Díaz, Vrabie [5]. Let $f:[0, T] \times L^{2}(\Gamma) \rightarrow L^{2}(\Gamma)$ and $g:[0, T] \rightarrow L^{2}(\Gamma)$ be defined by $f(t, u)(\sigma)=-\beta(t, u(\sigma))$ for $(t, u) \in[0, T] \times L^{2}(\Gamma)$ and a.e. for $\sigma \in \Gamma$, and respectively by $g(t)=\varphi(t)$ for each $t \in[0, T]$. Then, (9.1) can be rewritten as an abstract Cauchy problem of the form (8.1) in the Hilbert space $H$, with $\xi=u_{0}$, and Theorem 8.1 applies. An appeal to Theorem 3.1 shows that (i) holds. Since (ii) follows from well-known regularity results concerning elliptic equations along with the Green's Formula, the proof is complete.

Theorem 9.2. Assume that $g, \beta:[0, T] \times \mathbb{R} \rightarrow \mathbb{R}$ are continuous, have sublinear growth, $\eta \in B V\left([0, T] ; L^{2}(\Omega)\right)$, and $\psi \in B V\left([0, T] ; L^{2}(\Gamma)\right)$. Then, for each $u_{0}^{\Omega}$ in $L^{2}(\Omega)$ and $u_{0}^{\Gamma}$ in $L^{2}(\Gamma)$, there exists $T_{0} \in(0, T)$ such that the problem (9.2) has at least one solution $u:\left[0, T_{0}\right] \rightarrow L^{2}(\Omega)$ satisfying:

(i) for each $t \in\left[0, T_{0}\right)$ and each $s \in\left(0, T_{0}\right]$, we have

$$
\begin{cases}u(t+0)-u(t)=\eta(t+0)-\eta(t), & u_{\mid \Gamma}(t+0)-u_{\mid \Gamma}(t)=\psi(t+0)-\psi(t) \\ u(s)-u(s-0)=\eta(s)-\eta(s-0), & u_{\mid \Gamma}(s)-u_{\mid \Gamma}(s-0)=\psi(s)-\psi(s-0)\end{cases}
$$

(ii) for each interval $\mathbb{I}$ of absolute continuity of both functions $\eta$ and $\psi$ for which $\eta^{\prime} \in L^{2}\left(\mathbb{I} ; L^{2}(\Omega)\right)$ and $\psi^{\prime} \in L^{2}\left(\mathbb{I} ; L^{2}(\Gamma)\right)$, we have:

$u \in C\left(\mathbb{I} ; H^{1}(\Omega)\right) \cap W^{1,2}\left(\mathbb{I} ; L^{2}(\Omega)\right), u_{\mid \Gamma} \in C\left(\mathbb{I} ; H^{1 / 2}(\Gamma)\right) \cap W^{1,2}\left(\mathbb{I} ; L^{2}(\Gamma)\right)$, and $u, u_{\mid \Gamma}$ satisfy (9.2) a.e. on $\mathbb{I}$.

Proof. Take $H=L^{2}(\Omega) \times L^{2}(\Gamma)$ endowed with the usual inner product

$$
\langle(u, v),(\tilde{u}, \tilde{v})\rangle_{H}=\langle u, \tilde{u}\rangle_{L^{2}(\Omega)}+\langle v, \tilde{v}\rangle_{L^{2}(\Gamma)},
$$


for each $(u, v),(\tilde{u}, \tilde{v}) \in H$, and let us define the operator $A: D(A) \subseteq H \rightarrow H$ by

$$
D(A)=\left\{(u, v) \in L^{2}(\Omega) \times L^{2}(\Gamma) ; \Delta u \in L^{2}(\Omega), u_{\nu} \in L^{2}(\Gamma) \text { and } u_{\nu}=v \text { on } \Gamma\right\},
$$

and $A(u, v)=\left(\Delta u,-u_{\nu}\right)$ for each $(u, v) \in D(A)$. By Lemma 3.1 in BeJEnARU, DíAz, VRABIE [5], it follows that $A$ is a densely defined, self-adjoint operator which generates a compact analytic semigroup of contractions on $H$. Let us define $f:[0, T] \times L^{2}(\Omega) \times L^{2}(\Gamma) \rightarrow L^{2}(\Omega) \times L^{2}(\Gamma)$ and $g:[0, T] \rightarrow L^{2}(\Omega) \times L^{2}(\Gamma)$ by $f(t, u, v)(x)=-\left(\alpha\left(t, u(x), \beta(t, v(\sigma))\right.\right.$ for each $(t, u, v) \in[0, T] \times L^{2}(\Omega) \times L^{2}(\Gamma)$, a.e. for $x \in \Omega$ and a.e. for $\sigma \in \Gamma$, and respectively by $g(t)=(\eta(t), \psi(t))$ for each $t \in[0, T]$. Then, (9.2) can be rewritten as an abstract Cauchy problem of the form (8.1) in the Hilbert space $H$, with $\xi=\left(u_{0}^{\Omega}, u_{0}^{\Gamma}\right)$, and we are in the hypotheses of Theorem 8.1. The conclusion follows from Theorem 3.1 combined with well-known regularity results concerning parabolic equations.

\section{REFERENCES}

1. N. U. Ahmed, Some remarks on the dynamics of impulsive systems in Banach Spaces, J. of Dynamics of Continuous, Discrete and Impulsive Systems Ser. A Math. Anal. 8 (2001), 261-274.

2. P. Baras, J. C. Hassan, L. Veron, Compacité de l'opérateur définissant la solution d'une équation d'évolution non homogène, C. R. Acad. Sci. Paris Sér. I Math., 284(1977), 799-802. MR 55:3869

3. V. BARBU, Personal communication.

4. V. Barbu, Th. Precupanu, Convexity and Optimization in Banach Spaces, Second Edition, Editura Academiei Bucureşti, D. Reidel Publishing Company, 1986. MR 87k:49045

5. I. Bejenaru, J. I. Diaz, I. I. Vrabie, An abstract approximate controllability result and applications to elliptic and parabolic systems with dynamic boundary conditions, Electronic Journal of Differential Equations, 2001, no. 50, 1-19.

6. S. Bochner, A. E. TAYLOR, Linear functionals on certain spaces of abstractly-valued functions, Ann. of Math. 39(1938), 913-944.

7. H. BRÉZIS Opérateurs maximaux monotones et semi-groupes de contractions dans les espaces de Hilbert, Mathematics Studies 5, North-Holland, 1973. MR 50:1060

8. H. Brézis, A. Friedman, Nonlinear Parabolic Equations Involving Measures as Initial Conditions, J. Math. Pures et Appl. (9) 62(1983), 73-97. MR 84g:35093

9. J. Diestel, J. J. Uhl, JR., Vector Measures, American Mathematical Society, Mathematical Surveys, Number 15, 1977. MR 56:12216

10. N. Dunford, J. T. Schwartz, Linear Operators Part I: General Theory, Interscience Publishers, New York, London, 1958. MR 22:8302

11. K.-J. Engel, R. NAGEL, One-Parameter Semigroups for Linear Evolution Equations, Graduate Texts in Mathematics 194, Springer, 2000. MR 2001i:47075

12. L. M. Graves, The Theory of Functions of Real Variables, McGraw-Hill Book Company, Inc. New York and London, 1946. MR 8:319d

13. S. Gutman, Compact perturbations of $m$-accretive operators in general Banach spaces, SIAM J. Math. Anal. 13(1982), 789-800. MR 84d:34066

14. E. Hille, R. S. Phillips, Functional Analysis and Semi-Groups, Amer. Math. Soc. Colloquium Publications, Volume 31, Fourth Printing of Revised Edition, 1981. MR 19:664d (1st printing)

15. N. Hirano, Local existence theorems for nonlinear differential equations, SIAM J. Math. Anal., 14(1983), 117-125. MR 85b:34071

16. N. Hirano, Existence of periodic solutions for nonlinear evolution equations in Hilbert spaces, Proc. Amer. Math. Soc., 120 (1994), 185-192. MR 94b:34087

17. N. Hirano, N. Mizoguchi, Existence of periodic solutions for semilinear parabolic equations, Topology in Nonlinear Analysis, Banach Center Publications, 35 Institute of Mathematics Polish Academy of Sciences, 1996, 39-49. MR 98a:35005

18. C. S. HöNIG, Volterra Stieltjes-Integral Equations, Mathematics Studies Volume 16, NorthHolland/American Elsevier, 1975. MR 58:17705 
19. A. PAZY, A class of semi-linear equations of evolution, Israel. J. Math., 20(1975), 23-36. MR 51:11192

20. A. PAZY, Semigroups of Linear Operators and Applications to Partial Differential Equations, Springer-Verlag, Berlin, 1983. MR 85g:47061

21. J. Simon, Compact sets in the space $L^{p}(0, T ; X)$, Ann. Mat. Pura. Appl. (4) 146(1987), 65-96. MR 89c: 46055

22. N. Shioji, Local existence theorems for nonlinear differential equations and compactness of integral solutions in $L^{p}(0, T ; X)$, Nonlinear Anal., 26(1996), 799-811. MR 96k:34137

23. N. Shioji, Periodic Solutions for Nonlinear Evolution Equations in Banach Spaces, Funkcialaj Ekvacioj, 42(1999), 157-164. MR 2000k:34099

24. I. I. VRABie, The nonlinear version of Pazy's local existence theorem, Israel J. Math., 32, (1979), 225-235. MR 82a:47064

25. I. I. VRABie, Periodic solutions for nonlinear evolution equations in a Banach space, Proc. Amer. Math. Soc., 109(3)(1990), 653-661. MR 90k:34080

26. I. I. VRabie, A compactness criterion in $C(0, T ; X)$ for subsets of solutions of nonlinear evolution equations governed by accretive operators, Rend. Sem. Mat. Univers. Politecn. Torino, 45(1985), 149-157. MR 88g:34107

27. I. I. VRABIE, Compactness Methods for Nonlinear Evolutions, Second Edition, Pitman Monographs and Surveys in Pure and Applied Mathematics 75, John Wiley and Sons and Longman 1995. MR 96k:47116

28. I. I. VRABIE, Compactness in $L^{p}$ of the set of solutions to a nonlinear evolution equation, Qualitative problems for differential equations and control theory, C. CORDUNEANU Editor, World Scientific, 1995, 91-101. MR 96m:34125

Faculty of Mathematics, "Al. I. CuZA" University of Iași, Iaşi 6600, Romania

Current address: P. O. Box 180, Ro, Iş 1, Iaşi 6600, Romania

E-mail address: ivrabie@uaic.ro 